\title{
THE LOGIC AND TOPOLOGY OF KANT’S TEMPORAL CONTINUUM
}

\author{
RICCARDO PINOSIO AND MICHIEL VAN LAMBALGEN
}

\begin{abstract}
In this article we provide a mathematical model of Kant?s temporal continuum that satisfies the (not obviously consistent) synthetic a priori principles for time that Kant lists in the Critique of pure Reason (CPR), the Metaphysical Foundations of Natural Science (MFNS), the Opus Postumum and the notes and fragments published after his death. The continuum so obtained has some affinities with the Brouwerian continuum, but it also has 'infinitesimal intervals' consisting of nilpotent infinitesimals, which capture Kant's theory of rest and motion in MFNS. While constructing the model, we establish a concordance between the informal notions of Kant?s theory of the temporal continuum, and formal correlates to these notions in the mathematical theory. Our mathematical reconstruction of Kant?s theory of time allows us to understand what ?faculties and functions? must be in place for time to satisfy all the synthetic a priori principles for time mentioned. We have presented here a mathematically precise account of Kant?s transcendental argument for time in the CPR and of the relation between the categories, the synthetic a priori principles for time, and the unity of apperception; the most precise account of this relation to date. We focus our exposition on a mathematical analysis of Kant's informal terminology, but for reasons of space, most theorems are explained but not formally proven; formal proofs are available in (Pinosio, 2017).

The analysis presented in this paper is related to the more general project of developing a formalization of Kant's critical philosophy (Achourioti \& van Lambalgen, 2011). A formal approach can shed light on the most controversial concepts of Kant's theoretical philosophy, and is a valuable exegetical tool in its own right. However, we wish to make clear that mathematical formalization cannot displace traditional exegetical methods, but that it is rather an exegetical tool in its own right, which works best when it is coupled with a keen awareness of the subtleties involved in understanding the philosophical issues at hand. In this case, a virtuous ?hermeneutic circle? between mathematical formalization and philosophical discourse arises.
\end{abstract}

§1. Introduction: why formalize Kant's philosophy? The purpose of this paper is to provide a formalization of the theory of the temporal continuum developed by Immanuel Kant in his works of the critical period. ${ }^{1}$ Our formalization does not only shed light on some controversial interpretative issues, but also shows how Kant's philosophy can still be relevant for contemporary discussions on time and self-consciousness in philosophical logic, the philosophy of mind and cognitive science. This enterprise belongs to the more general project, which began in Achourioti and van Lambalgen (2011), of developing the mathematical theory of Kant's transcendental philosophy, and we believe it offers an example of how a really "scientific philosophy" in the Russellian

\footnotetext{
${ }^{1}$ We adopt the following system of abbreviations for Kant's works: CPR stands for The Critique of Pure Reason, MFNS stands for the Metaphysical Foundations of Natural Science, OP stands for the Opus Postumum, and $\mathrm{R}$ stands for the reflections. Passages from the CPR are cited with their usual numbering from the A and the B edition, while all other passages are cited according to their volume and page number from the Akademieausgabe edition of the collected works of Immanuel Kant (Kant, Holger, Gerresheim, Heidemann, \& Martin, 1908). The English translation of Kant's passages follows the Cambridge edition of the works of Immanuel Kant.
} 
sense (Russell, 1914) ought to proceed. The work in this paper is based on the material contained in Pinosio (2017), to which we shall often refer for the proofs of various results and for extended discussions that, for reasons of space, cannot be included here.

The reader is now probably wondering why anyone would want to formalize Kant's theory of time, let alone the whole of Kant's transcendental philosophy. Two sorts of objections immediately spring to mind. The Kant scholar will perhaps think that the applications of tools largely drawn from contemporary mathematics, which were hardly avaliable in Kant's time, is an anachronism or even a downright falsification and cannot deepen our understanding of Kant's thought. The logician, mathematician or philosopher of mind will perhaps doubt the fruitfulness of applying formal methods to Kant's exegesis, since Kant's philosophy is often regarded, not completely undeservedly, as plagued by ambiguities and riddled with hopelessly obscure jargon.

We disagree and instead believe that formalizing any systematic philosophy, and Kant's philosophy in particular, is fruitful and yields substantial gains. Only, this must be done with the utmost attention to exegetical accuracy; one must avoid the illusion, whose influence on many formal philosophers has been aptly denounced in (Rota, 1991), that philosophical problems can be resolved by merely writing down some axioms in a formal language, supposedly characterizing the basic informal philosophical notions at hand, and then proceed to uncritically take as the real content of the philosophical theory the consequences that can be computed from these axioms. On the contrary, we wish to make clear that mathematical formalization cannot displace traditional exegetical methods, but that it is rather an exegetical tool in its own right, which works best when it is coupled with a keen awareness of the subtleties involved in understanding the philosophical issues at hand. In this case, a virtuous "hermeneutic circle" between mathematical formalization and philosophical discourse arises.

A preliminary interpretation, on the basis of extant scholarship, of the systematic philosophy one is considering provides the first grounds on which a formalization can be developed. This preliminary interpretation, however, is not neutral to the aims of formalization; rather, the goal of developing "formal correlates" to the central concepts of the system influences the interpretation of their content, so that the aim of formalization drives the interpretation itself. Every act of formalization of an informal philosophical text is then an act of interpretation in its own right and is dependent on a preliminary exegesis of the concepts involved, so that the choice of the mathematical formalism already embodies an interpretational stance: it implies that certain aspects of the system, which are considered as fundamental, will be captured in the formalization, while others, which are considered as secondary, will be abstracted away.

Next, however, one evaluates the exegetical accuracy of the formalization; textual evidence and philosophical analysis can prompt its revision if certain parts are not deemed accurate, but at the same time the formalization itself can suggest new interpretative possibilities that might not be immediately apparent without the use of formal tools. Whenever the formalization of certain concepts throws a specific light on the content of other concepts, it can prompt a revision of the interpretative stance towards revising, extending or consolidating the formal theory.

The general features of this "virtuous circle" between interpretation and formalization, and its usefulness in relation to understanding any systematic philosophy, are discussed in more detail in Pinosio (2017). We shall here only mention, very briefly, how 
it can improve our understanding of Kant's transcendental philosophy and Kant's theory of time in particular, and refer the reader to Pinosio (2017) for a more detailed justification.

The most important advantage of formalization is that it helps to clarify the structure of the intricate web of concepts of Kant's philosophy and of Kant's argumentation, so that its evaluation is simplified and a more precise comparison of competing interpretations is made possible. This is seen most clearly in the case of Kant's theory of time; while certainly crucial for the general argument of the first Critique, this theory is not obviously consistent, and its most difficult passages have given rise to multiple competing interpretations. Indeed, there have been fierce debates on how to interpret even some of the most fundamental concepts of Kant's theory of time, such as the distinction, at B161n, between time as form and as formal intuition. We aim to show here that the resources of formal logic can be used effectively to shed light on these matters.

Beyond strictly exegetical considerations, however, we also believe that Kant's theoretical philosophy is relevant to contemporary discussions in the philosophy of space and time, in the foundations of mathematics and physics, and in the cognitive sciences. Indeed, there is an increasing awareness that Kant's treatment of space, time and causality can still be a rich source of ideas for cognitive science (Northoff, 2012; Dehaene \& Brannon, 2011; Palmer, 2008; Palmer \& Lynch, 2010), and we hope that a mathematical model that captures the main aspects of Kant's thought on these matters can help to make this relevance explicit. While we do not have the space here to go into too much detail about this (but see section 3.9 in Pinosio (2017)), we shall provide some remarks relevant to cognitive science in the next section.

The structure of the paper is as follows. In section 2 we discuss the philosophical foundations of the formal theory that we develop in this paper. In section 3 we provide an axiom system for Kant's intuition of time and justify its exegetical accuracy. In sections 5 to 7 we discuss the construction of Kantian boundaries or instants in time, which is crucial to understand Kant's notion of infinite divisibility of time. In section 8 we provide a formalization of the self-affection of inner sense through the figurative synthesis using inverse systems and inverse limits. In section 9 the treatment of the paper culminates with a formalization of the Kantian continuum arising from infinite divisibility, while in section 10 we discuss specifically duration and the external representation of time as a line. We conclude the paper in section 11 with an overview of what has been achieved.

\$2. Philosophical foundations: time and the imagination. We provide here the bare minimum of discussion of Kant's theory of space and time that is needed to understand the point of the formalization presented in the following sections. While a certain amount of Kantian jargon is unavoidable, we have attempted to explain it as clearly as possible and to present this material in the simplest possible way for readers who do not know much about Kant. This also means that we cannot go here into a detailed and exegetically fully rigorous justification of our interpretation; but see (Pinosio, 2017) for more detail.

2.1. The properties of time. The CPR begins with a section called the Transcendental Aesthetic, ${ }^{2}$ where Kant claims that space and time are not concepts but intuitions

\footnotetext{
${ }^{2}$ Henceforth TA.
} 
acquired a priori and that they satisfy various properties that can be termed synthetic a priori principles for space and time (some of these principles, however, are only discussed after the TA). Here, the term "a priori" indicates that these intuitions are acquired, and its necessary properties are established, without recourse to any particular sensory experience. We hasten to add that this does not mean that they are in any way innate; on the contrary, Kant speaks of the original acquisition of space and time through acquaintance with sensory data (Ak. 8:223; see also (Longuenesse, 1998, p.252). Moreover, the notion of necessity that occurs here is to be interpreted as "satisfied in every possible experience"; in other words, these properties are claimed to be satsified by the intuition of time of any possible cognizing subject that is sufficiently similar to us. These principles are also synthetic because they are not conceptual truths: they cannot be obtained through conceptual analysis of the concept "time", as it were. These synthetic a priori principles are:

(1) time is infinite: every part of time is part of the unbounded, all-encompassing time

(2) time is divisible to infinity

(3) time is linear: "time has only one dimension; different times are not simultaneous, but successive" (A31/B47)

(4) time is not composed out of infinitely many parts, rather these are only limitations of the whole antecedently given time: "different times are only parts of one and the same time" (A31-31/B47)

(5) time is unique, i.e., there exists only one time

(6) time is continuous and connected, i.e., time has no "jumps" with "empty time" in-between

(7) time supports the assignment of a "magnitude of duration" to events, which can be infinitesimally short

The properties of infinity and infinite divisibility deserve a special discussion. Kant's definition of infinity is usually formulated in "mereological" terms: a magnitude is infinite if it is such that "every magnitude of the same type is only a part of it" (KT 20:419). In particular, he claims that space and time are "infinitely given magnitudes", since every extent of time must be part of an infinite, all-encompassing time. Now, some commentators infer from this that Kant takes space and time to be actual infinities, and contrast the infinity of space and time as intuitions with the merely potential infinity of geometrical spaces, e.g. the geometrical line, whose construction is a process that proceeds in infinitum.

This characterization, however, is misleading, since the contemporary notion of actual infinity involves a "completed" infinity of distinct units, which Kant would not accept in relation to space and time because infinite collections of units cannot be "surveyed" or "apprehended". Rather, we have argued (Pinosio, 2017) that when Kant says that space and time are "infinitely given" magnitudes he means that when the subject constructs a geometric line in intuition it becomes aware of the unboundedness of the

\footnotetext{
${ }^{3}$ More explicitly:

Spatium est quantum, sed non compositum. For space does not arise through the positing of its parts, but the parts are only possible through space; likewise with time. The parts may well be considered abstrahendo a caeteris, but cannot be conceived removendo caetera; they can therefore be distinguished, but not separated, and the divisio non est realis, sed logica. (R4425)
} 
progress in this construction, of its "indefinite extensibility". This awareness is then immediately the consciousness of an infinite object because, as far as space and time are concerned, what is "possible" is also "actual" (R4515, 17:579 and R6290, 18:559), that is, for space and time consciousness of a potential iterative construction is immediately consciousness of its limit product. Infinite divisibility is to be understood in a similar fashion, as "potential to actual": any given stretch of time can be potentially divided to infinity, but we are also warranted in considering the infinite completion of this process.

Let us return to the synthetic a priori principles. These are also "transcendental" since they pertain to Kant's investigations, in his works of the critical period, into the "conditions of possibility" of experience. This investigation often assumes the form of a transcendental argument, which is perhaps the most important innovation introduced by Kant into philosophical discourse. In a transcendental argument one first takes a supposedly manifest feature of experience as granted, and then one argues that certain less evident conditions must be in place for this feature to be possible; moreover, for the argument to be convincing one has to argue that these conditions are not only sufficient, but also necessary to justify the manifest feature of experience at hand.

The starting point of our formalization of Kant's theory of time is that Kant's whole discussion of space and time in the CPR assumes the form of a long transcendental argument. Indeed, the transcendental a priori principles for space and time announced in the TA might seem to come out of the blue with little to no justification; it might seem as if Kant believes that we have a direct "introspective" access to the properties of space and time, and that these come into being already formed and accessible to our mind without the need of any processing of sensory impressions. Indeed, many commentators, who are generally termed "nonconceptualists", have defended an interpretation along similar lines. ${ }^{4}$ We argue, however, that this interpretation overlooks the fundamental fact that the definitions of space and time that are given in the TA must not be conceived as final, since, in Kant's words:

[ ... ] in philosophy one must not imitate mathematics in putting the definitions first, unless perhaps as a mere experiment. For since they are analyses of given concepts, these concepts, though perhaps only still confused, come first, and the incomplete exposition precedes the complete one, so that we can often infer much from some marks that we have drawn from an as yet uncompleted analysis before we have arrived at a complete exposition, i.e., at a definition; in a word, it follows that in philosophy the definition, as distinctness is made precise, must conclude rather than begin the work. (A731/B759)

If we apply this principle to Kant's own philosophy, this means that Kant's discussion of space and time in the TA marks only a first beginning towards an understanding of these concepts, and what comes afterwards, in particular the chapter on the Transcendental Deduction of the Categories, ${ }^{5}$ must necessary prompt a "re-reading" (Longuenesse, 1998, p. 299) and clarification of the TA in light of the deeper discussions there contained. But what form does such a "re-reading" take? We argued in that it takes the form of a transcendental argument, and, although as we remarked we cannot discuss such matters extensively in this paper, we shall briefly mention the main lines of the

${ }^{4}$ See Onof and Schulting (2015) for a recent review of the discussion and a defense of nonconceptualism about space and time.

${ }^{5}$ Henceforth TD. 
argument, so as to provide the reader with an outline of the philosophical foundations of the formalization presented here.

2.2. Three questions on the intuition of time. A good place to start our outline of Kant's theory of time are the "General remarks on the Transcendental Aesthetic" in the $\mathrm{CPR}$, where we first find a definition of the form of intuition of space and time:

[ ... ] everything in our cognition that belongs to intuition [ $\ldots$ ] contains nothing but mere relations [Verhältnis], of places in one intuition (extension), alteration of places (motion), and laws in accordance with which this alteration is determined (moving forces) [ ... ] it is not merely that the representations of outer sense make up the proper material with which we occupy our mind, but also the time in which we place these representations [ ... ] already contains relations of succession, of simultaneity, and of that which is simultaneous with succession (of that which persists). Now that which, as representation, can precede any act of thinking something is intuition and, if it contains nothing but relations, it is the form of intuition, which, since it does not represent anything except insofar as something is posited in the mind, can be nothing other than the way in which the mind is affected by its own activity [ . . ] (CPR B68, our emphasis).

The form of the intuition of time consists only in relations of succession, of simultaneity and of persistence, according to which all our representations must be ordered, ${ }^{6}$ but which itself precedes the consciousness of these representations in experience a priori. Similarly, in the TA Kant characterizes the form of intuition as "that which so determines the manifold of appearance that it allows of being ordered" (A20/B34). Now, three substantial problems arise for the logician in understanding this passage.

First, what are these relations of succession? Various possible temporal relations spring immediately to mind: temporal precedence, temporal overlap, simultaneity, and so forth. Kant's definition does not seem to specify whether all these relations, or only some of them, are salient.

Second, what exactly are the relata in question? Are they point-like instants of some sort, are they events or durations having some breadth or extension? Do they represent empirical events, such as the collision of two bodies in space, or are they something more "fundamental", i.e. a priori?

Third, how is one to construe the obscure claim that the form of intuition is nothing other than "the way in which the mind is affected by its own activity"?

We shall consider each of these questions in turn.

2.3. The Kantian continuum. We begin with the second questions: what are the relata of time as the form of intuition? We can obtain a first answer by considering Kant's thought on the concept of the continuum. Indeed, according to Kant the continuum is not constructed in terms of a set of dimensionless points, as in the construction that is standard in contemporary mathematics. Rather, it is given as a whole, where parts of time - which are themselves times or "subcontinua" - can be individuated by delimiting them with points or boundaries, which are then only "limitations" of times and supervene on extended parts of time:

The property of magnitudes on account of which no part of them is the smallest (no part is simple) is called their continuity. Space and time are quanta continua because no part of

\footnotetext{
${ }^{6}$ Kant speaks here only of ordering the representations of outer sense in time; however at A34 he remarks that all representations are ordered in time, not only those of outer sense, but also those of inner sense.
} 
them can be given except as enclosed between boundaries (points and instants), thus only in such a way that this part is again a space or a time. Space therefore consists only of spaces, time of times. Points and instants are only boundaries, i.e., mere places of their limitation; but places always presuppose those intuitions that limit or determine them, and from mere places, as components that could be given prior to space or time, neither space nor time can be composed. Magnitudes of this sort can also be called flowing, since the synthesis (of the productive imagination) in their generation is a progress in time, the continuity of which is customarily designated by the expression "flowing" ("elapsing"). All appearances whatsoever are accordingly continuous magnitudes [ . . . ] (A16970/B211-2, our emphasis $)^{7}$

It is interesting that Kant's considerations on points and instants echo Aristotle's claim that "time is not composed of indivisible nows any more than any other magnitude is composed of indivisibles" (Physics, 239b 5) and that:

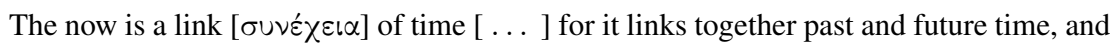
in its general character of "limit" it is at once the beginning of time to come and the end of time past. ${ }^{8}$ (Physics, 222a 10-20, our emphasis)

Kant's conception of instants differs from Aristotle, however, in considering the possibility that instants are extended rather than point-like:

(4) are two different states separated by a time that is not filled through a continuous series of alterations[?] The instant in time can be filled, but in such a way that no time-series is indicated. All parts of time are in turn times. The instant. Continuity. (R4756, 17:700)

The occurrence of can in this passage is important, since it betrays Kant's modal conception of potential infinity and infinite divisibility that we shall model in the sequel using inverse systems. In any case, from these observations we can reliably infer that the relata of time as the form of intuition are parts of time, having some breadth or extension. It is these parts of time that are related according to relations of succession, simultaneity and persistence.

2.4. Relations of succession. In the previous section we showed that the form of the intuition of time consists of "relations of succession" that hold among "extended events". We must now investigate what sort of "relations of succession" among these events one ought to consider. Since Kant is never really explicit about which spatial or temporal relations are primitive, we must attempt to infer this from the consideration of his whole system.

Let us first examine those relations that are related to coexistence in time. A good candidate in this respect is the binary relation $O$ with intuitive meaning: $a O b$ if event $a$ "temporally overlaps" with event $b$. Evidence for the salience of the overlap relation can be found in the third analogy at B257, where Kant glosses simultaneity in terms of temporal overlap. If I first observe $A$ and then observe $B$ and then observe $A$ again I determine my representations of $A$ and $B$ in a relation of temporal overlap, so that they are cognized as being simultaneous in the sense that since they overlap there is a time in which they both exist, which is Kant's definition of simultaneity.

\footnotetext{
${ }^{7}$ This passage shows that Kant's theory of time is an instance of a more general tradition of thought on the problem of the continuum that we might term "continuist" in opposition of the standard, "Cantorean" conception; see Pinosio, 2017, p. 19 for a more in-depth discussion of these matters.

${ }^{8}$ See also Aquinas' commentary on Aristotle's Physics, Bk. 6.861.
} 
Along with relations encoding coexistence in time we must have relations encoding temporal order among events. Here various choices are possible, each corresponding to a subset of the set of fundamental relations among events identified by Allen (). In light of the work in philosophical logic on the construction of time from events (Russell, 1936; Thomason, 1984, 1989; Van Benthem, 2013) one might recur to the binary relation $P$ of complete precedence, with intuitive meaning: $a P b$ if event $a$ ends before event $b$ begins, i.e., $a$ "completely precedes" $b$. The salience of this relation seems at first supported by Kant's emphasis on relations of "succession". However, this cannot be quite right in view of Kant's treatment of causality. Indeed, Kant holds that a cause does not have to completely precede its effect, but that, on the contrary, it can be simultaneous with its effect (A202/B248). Hence, complete precedence is too strong. We then take as primitive the binary relations $\mathbf{R}_{-}, \mathbf{R}_{+}$, with intuitive meaning: $a \mathbf{R}_{-} b$ if event $a$ ends before or simultaneously with event $b$ ( $a$ is in the past of $b$ or $b$ does not end before $a$ ), $a \mathbf{R}_{+} b$ if event $a$ begins after event $b$ or simultaneously with it ( $a$ is in the future of $b$ or $b$ does not begin after $a$ ). These relations are salient because the discussion at A202/B248 makes clear that to treat Kant's notion of causality formally one must be able to compare events with respect to their beginning and their end.

We also define a binary relation $\preceq$ of "covering" as $a \preceq b$ iff $a \mathbf{R}_{-} b \wedge a \mathbf{R}_{+} b \wedge$ $O(a, b)$. It is useful to think of the covering relation among events as a relation of temporal encompassment: given two events $a, b$ of description of spaces, $a$ covers $b$ if the temporal extent of $a$ "encompasses" the temporal extent of $b{ }^{9}$

In what follows, we shall also add two partial binary operations $\oplus, \ominus$ between events, whose introduction will be justified in terms of the category of causality. In particular, given events $a, b$, we shall let $a \oplus b$ be an event representing "the part of $a$ that can be causally influenced by $b$ ", and let $a \ominus b$ be an event representing "the part of $a$ that can causally influence $b$ "; but see section 3 for more detail.

2.5. Events of self-affection through attentional motion. We have now provided an aswer to two of the questions we posed at the beginning: we know what the relata of time as the form of intuition are and we know what relations of succession among events we should consider.

The reader, however, will now most likely be puzzled: what are these events exactly, that is, what do they represent? In order to answer this question we must address what we have up to now left untouched, that is, we must understand what Kant means when he says that "the mind is affected by its own activity".

To understand this obscure remark we must backtrack a little and consider in what sense Kant's theory of space and time in the CPR constitutes a transcendental argument. We remarked before that Kant's discussion of the synthetic a priori principles for space and time in the TA seems to come almost out of the blue. Now, the synthetic a priori principles announced in the TA are indeed obtained through introspection, but they are followed by an investigation - mostly carried out in the TD - into the faculties and functions that must necessarily be in place so that the intuitions of space and time do indeed satisfy such properties, and this comprises in turn an investigation into the faculties and functions that are responsible for the acquisition, albeit a priori, of these intuitions. Our interpretation (Pinosio, 2017) of Kant's account of the acquisition of the representation

${ }^{9} \mathrm{Nicod}$ (Nicod, 2014) emphasizes this relation of "encompassment", which he takes as a primitive relation. Kant himself explicitly recognizes this relation as salient at (R4756, 17:701). 
of time is quite modern, since it is in surprising agreement with current results in cognitive science showing that our representation of time is very much dependent on its external representation in space as a line (Casasanto \& Boroditsky, 2008; Casasanto, Fotakopoulou, \& Boroditsky, 2010; Carelli \& Forman, 2012).

In particular, space and time are acquired when the subject "affects itself" through the description of a space in outer sense, where this "description of a space" can be thought of as the serial movement of the attentional focus, which affects both the "inner" and the "outer" sense. ${ }^{10}$ In the "drawing of a line in space" the subject produces impressions for itself, it arranges them in space and time and obtains a priori access to the action through which it apprehends an empirical manifold in general, that is, through which it effects a synopsis and unification of sensory impressions in the concept of an object. Moreover, in providing these impressions and in becoming aware of the identity of the action of synthesis of these impressions, the subject makes itself into the original object of apprehension and thereby structures itself spatiotemporally; it represents itself as an object in spatiotemporal relations with other objects, which is the source of the subject's self-consciousness, as it becomes aware of the succession of its representations and therefore of the succession of itself in time:

Our sensible intuition is, initially, not perception (empirical representation with consciousness), for a principle of positing oneself and of becoming conscious of this position precedes it; and the form[s] of this positing of the manifold, as thoroughly combined, are the pure intuitions, which are called space and time $[\ldots]$ (22:420)

When the subject affects itself through motion of the attentional focus, for which it must " arouse [the sensations] and connect them synthetically, hence affect itself" (R6349, 18:674), it thereby becomes conscious of the universal forms of any such act, and these are space and time.

Kant's term for this self-affection through motion of the attentional focus is the figurative synthesis of the imagination:

[ ... ] we cannot think of a circle without describing it [ . . ] we cannot even represent time without, in drawing a straight line (which is to be the external figurative representation of time), attending merely to the action of the synthesis of the manifold through which we successively determine the inner sense, and thereby attending to the succession of this determination in inner sense. Motion, as action of the subject [ . . . ] consequently the synthesis of the manifold in space, if we abstract from this manifold in space and attend solely to the action in accordance with which we determine the form of inner sense, first produces the concept of succession at all $[\ldots]^{11}$ (B154-B155)

\footnotetext{
${ }^{10}$ The reader might think that we are forcing the text here, but this is not the case: Kant himself speaks of "attention" in relation to the self-affection in the description of a space at B156-57 and (20:270) (Pinosio, 2017, p. 53).

${ }^{11}$ As a footnote to this passage we find:

Motion of an object in space does not belong in a pure science, thus also not in geometry; for that something is movable cannot be cognized a priori but only through experience. But motion, as description of a space, is a pure act of the successive synthesis of the manifold in outer intuition in general through productive imagination, and belongs not only to geometry but even to transcendental philosophy
} 
Thus, we can have conscious representations of space, time and their properties only if we delineate trajectories in outer sense and focus on the form of how we are affected. ${ }^{12}$ It is important to emphasize that it is really trajectories that are at issue here rather than mere geometrical constructions, since motion, as the original act of the description of a space, combines both inner and outer intuition, time and space ${ }^{13}$; thus, the description of a space is really a spatio-temporal act, and the subject can either focus on the outer manifold which is produced or focus on the determination of the inner sense in producing it. It thereby becomes conscious of the necessary properties of its forms of outer and inner sense, respectively.

This figurative synthesis, moreover, is constrained by the categories, which are the logical functions of judgments in Kant's transcendental logic (Achourioti \& van Lambalgen, 2011) and the source of any possible connection of empirical appearances that is thought as objective. The role of the categories in relation to the figurative synthesis is important, because this synthesis pertains to the faculty of the imagination, so that in the absence of any constraint from the understanding on how it is to proceed it could produce and connect the sensory impressions in whatever possible form; Kant's example of this are dreams, where the imagination runs unruly and the usual properties of space and time do not apply. Moreover, if we are to become conscious of the form of any possible act of apprehension of an object $a$ priori we must not only provide sensory data to ourselves via movement of the attentional focus, but also make sure that the connection we effect in this manifold of sensory data would allow for it to be thought as an object, for which it must conform to the sensible conditions ensuring that it can be reflected according to the functions of judgment, namely, the categories.

The ultimate foundation of the categories as logical functions of judgments is, however, the principle of the unity of apperception, or unity of consciousness, which according to Kant is contained in all the categories. This principle states that the identity of the consciousness that accompanies distinct representations (e.g., the representation of a color and of a shape that is of that color) is a fundamental feature of the subject, but this feature can only be ensured through a necessary combination or connection between representations, which is expressed in the judgment, so that the identity of the consciousness accompanying many different representations can be thought only if these can be ordered and combined into an objective whole according to the categories. Thus, time and space are a product of the figurative synthesis in agreement with the categories and ultimately with the unity of consciousness, and the figurative synthesis and its properties are necessary to show that the synthetic a priori principles of time apply to any possible experience.

${ }^{12}$ The following passage is even more explicit in this sense:

We need space in order to construct time, and thus determine the latter by means of the former $[\ldots]$ in the representation of space we must be conscious of ourselves as being affected by outer things. We do not cognize this by means of an inference, rather it lies in the way in which we affect ourselves in order to construct time as the mere form of the representation of our inner state, for which something other, not belonging to this inner state, must still always be given (i.e., something outer, the construction of which at the same time contains the intuition of time and lies at its ground). (R6311, 18:613)

the construction of a line "contains the intuition of time and lies at its ground" since if I produce a trajectory and focus on the successive determination of the inner sense I first produce the intuition of time.

${ }^{13}$ Kant remarks this explicitly in the phoronomy section of the MFNS ([489]) and at 22:440 of the OP, where he says that motion combines both the outer intuition (space) and the inner intuition (time) in one. 
We infer from this discussion that the "extended events" that are the relata of the relations of succession are actually a priori events of self-affection, that is, they represent acts of descriptions of a given trajectory in a given time. The next section will clarify this notion further. We note, however, that while the purpose of this work is to provide a formal theory that elucidates and supports the interpretation which we have briefly outlined, and that also sheds light on various other aspects of Kant's theory of time such as its relation to Aristotle's theory of time in the Physics, many scholars would judge the idea that space and time in the TA are produced by the figurative synthesis wrong. They would rather hold that space and time as intuitions are given without the need of any "synthesis" or "process" depending on the understanding, almost as if we had direct intuitive access to space and time. We restrict ourselves here to pointing out that Kant's quote in the introduction suggests that any interpretation that takes the treatment of space and time in the TA as final is misplaced, and refer the reader to the discussion of these matters in Pinosio (2017).

2.6. The general form of an act of description. We can gain additional insight on the notion of self-affection through a priori motion by examining what the general form of an act of self-affection in the description of a space is. Consider the simplest case: a point of focal attention that moves periodically between to locations in space, thereby describing a line segment. This act of description can be represented by the diagram in Figure 1.

The idea of the diagram is the following. The vertical axis represents the inner sense, the horizontal axis represents the outer sense. The portions of the curve labelled $A$ to $E$ represent acts of description of spaces in outer sense, through which certain spaces are described in certain times and the inner sense is thereby affected successively.

In particular, different acts are individuated by changes to the state of motion of the point, so that, e.g., $A, C, E$ are states of approximate rest of the moving point, and $B, D$ are states of approximate constant velocity. Of course, the word "approximate" is essential here, as the diagram in Figure 1 represents only a first approximation in the analysis of the act of description. The moving point is really only at rest at a moment of infinitesimal duration, and it undergoes a constant acceleration before reaching the constant velocity. However, this is a central point in Kant's analysis of motion, which is closely tied to his theory of the spatial and temporal continuum: a motion is first given as a coarse whole, which can then be analyzed further by distinguishing finer and finer submotions identified by changes of state of the movable point determined more and more precisely, yielding a sequence of refinements. We shall provide a way to formalize this process in section 9.

The dashed part of the curve indicates that the motion under consideration is periodic, as the point keeps moving back and forth between the two locations; this sort of motion is of importance as Kant states that the perception of simultaneity occurs whenever I become aware that I can apprehend the manifold in different orders "as many times as I want", an act which he terms the comprehension of the manifold and that is an essential part of the apprehension of an object, since without it no objective determination of temporal relations is possible. For a given act of description, say $A$, we denote with $\pi_{t}(A)$ the time in which the space is described, which is really a representation with consciousness in inner sense of an object of outer sense, a space which we might denote with $\pi_{s}(A)$; everything which we encounter in our consciousness is a representation in inner sense of a manifold in outer sense. 


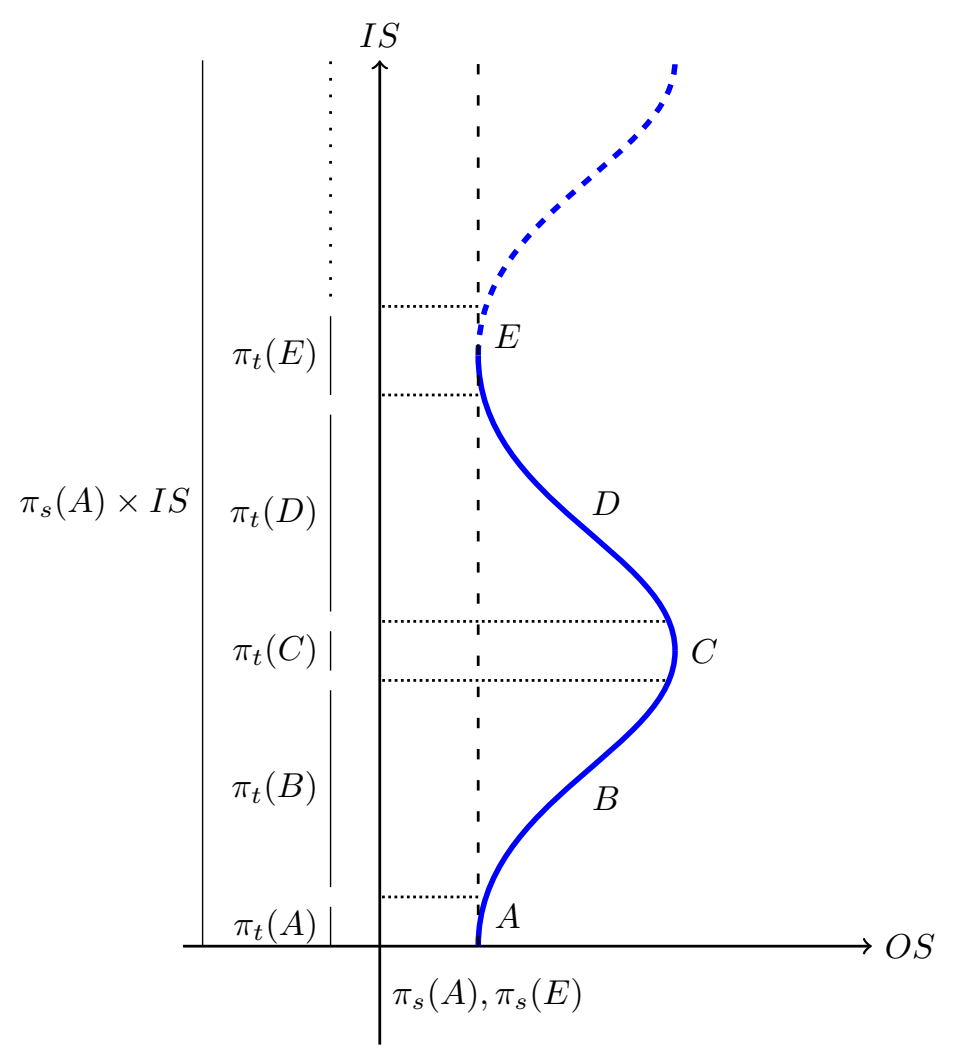

FIGURE 1. An act of description

Now, if the spaces I synthesize in outer sense did not persist through time, but disappeared as soon as the figurative synthesis moved to the synthesis of other spaces, then I would not be able to cognize, e.g., that $\pi_{s}(A)$ coexists in time with $\pi_{s}(C)$. For then $\pi_{s}(E)$ would be represented as a space wholly different than $\pi_{s}(A)$, a space synthesized anew, and I would be left with a series of synthesis of always different spaces, so that overlap, and hence simultaneity, could never be perceived. Moreover, according to Kant (R5348, 18:158), without the persistence of the spaces that I synthesize in outer sense I could not cognize the succession of the events of self-affection themselves, since to be conscious of a succession in inner sense one must be conscious that one has perceived the same thing at different times. Thus without persistence of spaces there is no cognition of identity through time, and I could not cognize even the difference between, say, $\pi_{t}(A)$ and $\pi_{t}(E)$, let alone that they stand in an objective succession. Thus, the representation of the persistence of the spaces synthesized in outer sense is necessary for the cognition of simultaneity and succession, so that the construction of time depends on space. In the diagram, the dashed vertical line and the vertical line labelled $\pi_{s}(A) \times I S$ denote the representation of the persistence of $\pi_{s}(A)$, against which the succession of, e.g., $\pi_{t}(A)$ and $\pi_{t}(E)$ is cognized. Still, it is only because I cognize simultaneity through the construction of geometrical spaces that I can consciously represent space 
as persistent; hence the representation of space itself depends on time. In other words, in the construction of geometrical spaces through motion I "simultaneously" institute space and time as intuitions.

The Diagram in Figure 1 also highlights the role that infinitesimals play with respect to the notion of the description of a space, since according to Kant a point is at rest at a location, say at $\pi_{s}(E)$, if it has infinitesimal velocity, i.e. a velocity that is smaller than any given velocity, and not null velocity. Thus, while discussing the motion of an object projected upward from a point $A$, and reversing direction of motion at point $B$ under the influence of gravity, Kant states that:

[ ... ] the speed at point $B$ is not completely diminished, but only to a degree that is smaller than any given speed. With this speed, therefore, the body would, if it were to be viewed always as still rising [ . . . ] uniformly traverse with a mere moment of speed (the resistance of gravity here being set aside) a space smaller that any given space in any given time no matter how large. (4:486)

Hence, the events of self-affection labelled $\pi_{t}(A), \pi_{t}(C), \pi_{t}(E)$ represent the point at rest, i.e., moving with infinitesimal velocity, and are to be conceived of as representing clusters of infinitesimals. We shall return on the formalization of these infinitesimals in section 10 .

Note finally that the act of description, since it is produced by the figurative synthesis, must be constrained by the categories as rules for the objective determination of inner and outer sense. Thus the representation of the permanence of $\pi_{s}(A)$, the event $\pi_{s}(A) \times$ $I S$, depends on the category of substance, while the representation of the objective succession of $\pi_{t}(A)$ and $\pi_{t}(E)$ depends on the category of cause, which determines the imagination so that the latter event succeeds the former. We shall have more to say on the role of the categories in the figurative synthesis in the following section.

§3. An axiom system for Kant's intuition of time. In the previous sections we have seen that the "pure events" that are the relata of the form of intuition in the TA are events of self affection in the description of spaces by the figurative synthesis, through which the manifold originally provided by the sensibility is structured in spatiotemporal relations. There must be certain axioms, however, ensuring that the relations produced by the figurative synthesis satisfy the properties of time in the TA. That is, without axioms that constrain the action of the figurative synthesis this would be unruly, and could determine the relations of time in a way that is unlike that described by Kant in the TA. Not even the simplest properties of time - e.g., transitivity and linearity of $\mathrm{R}_{+}, \mathrm{R}_{-}$- could then be ensured.

These axioms, however, effectively limit what counts as a possible temporal form of an experience, since they rule out certain forms; e.g., branching time. Thus, they represent a priori constraints on the action of the figurative synthesis, and as such they must be grounded in the categories, as basic functions for the objective combination of representations.

To capture these considerations formally we start with finite sets of events of selfaffection which are ordered according to the temporal relations presented in section 2.4. Every finite set of events represents a manifold that may be produced by the figurative synthesis through finitely many acts of description, such as acts $A$ to $E$ in Figure 1 . We consider finite sets because, as we discussed in section 1 , the infinity of time consists in the consciousness of the unboundedness of any act of apprehension; starting with 
actually infinite sets of events would run contrary to Kant's dictum that an actual infinity of distinct units cannot be intuited, and so cannot be a possible experience. Now, on these sets of events we shall impose axioms of temporal order that can be derived from the analysis in section 2.6, and justify them in terms of the categories.

3.1. The axioms of temporal order. We consider the following axioms of temporal order:

DEFINITION 1. A first-order structure in the signature $\left(\mathrm{R}_{+}, \mathrm{R}_{-}, O ; \preceq ; \oplus, \ominus\right)$ is an event structure if it is a model of the following axioms:

(1) $a \preceq b \leftrightarrow a \mathbf{R}_{+} b \wedge a \mathbf{R}_{-} b$ (Explicit definition of $\left.\preceq\right)^{14}$

(2) $a \overline{O b} \rightarrow b O a$ (symmetry of $O$ )

(3) $a O a$ (reflexivity of $O$ )

(4) $c O b \wedge c \mathrm{R}_{+} a \wedge b \mathrm{R}_{-} a \rightarrow a O b$ (condition for overlap)

(5) $a \mathrm{R}_{+} b \wedge b \mathrm{R}_{+} c \rightarrow a \mathrm{R}_{+} c$ (Transitivity of order)

(6) $a O c \wedge c O b \wedge c \mathrm{R}_{+} b \wedge c \mathrm{R}_{+} a \rightarrow a O b$ (conditional transitivity of $O$ )

(7) $b \mathrm{R}_{+} a \vee a \mathrm{R}_{+} b$ (linearity)

(8) $\exists c(a \preceq c \wedge b \preceq c)$ (covering axiom)

(9) Partial binary operations $(\oplus, \ominus)$ on events (we write $a \oplus b=y$ for $\oplus(y, a, b)$ ).

(a) $a \oplus b=y \wedge a \oplus b=z \rightarrow y=z$ (functionality)

(b) $a \mathrm{R}_{+} b \vee a O b \leftrightarrow \exists y(y=a \oplus b)$ (explicit domain of definition)

(c) $a \oplus b \mathbf{R}_{+} b$

(d) $a \oplus b \mathrm{R}_{+} a$

(e) $a \mathrm{R}_{-} a \oplus b$

(f) $a \oplus b \mathrm{R}_{-} a$

(g) $a \mathrm{R}_{+} b \rightarrow a \mathrm{R}_{+} a \oplus b$

(h) $b \mathrm{R}_{+} a \rightarrow b \mathrm{R}_{+} a \oplus b$

(i) $a \oplus a=a$

(j) $(a \oplus b) \oplus c=(a \oplus c) \oplus b$

(k) $(a \oplus b) \oplus b=a \oplus b$

(l) $(a \oplus b) \ominus c=(a \ominus c) \oplus b$

(10) Any sentence $\phi$ obtained from the above axioms by simultaneously replacing $\mathrm{R}_{-}$ for $\mathbf{R}_{+}, \mathbf{R}_{+}$for $\mathbf{R}_{-}$, $\ominus$ for $\oplus$ and $\oplus$ for $\ominus$ (substitution principle)

Note that free variables are understood as universally quantified, and moreover that the axioms of group (9) are actually of the form

$$
\exists y(a \oplus b=y) \rightarrow \Phi
$$

All the intuitive properties of $O, \mathrm{R}_{+}, \mathrm{R}_{-}$follow from the axioms above; see Pinosio (2017, p. 96). In what follows we shall sometimes use symmetry and reflexivity of $O$ without mention, and make use of the following abbreviations:

DEFINITION 2. Let $\mathcal{W}$ be an event structure. For convenience we define the following abbreviations:

- $a \equiv_{-} b$ if $a \mathrm{R}_{-} b, b \mathrm{R}_{-} a$

- $a \equiv \equiv_{+} b$ if $a \mathrm{R}_{+} b, b \mathrm{R}_{+} a$

- $a \equiv b$ if $a \preceq b, b \preceq a$

${ }^{14} \mathrm{We}$ do not need to require that $a O b$, since this follows from symmetry of $O$ and axiom (4) 
- $a \mathbb{O} b$ if $\neg(a O b)$

- $a \mathbb{R}_{-} b$ if $\neg\left(a \mathrm{R}_{-} b\right)$

- $a \mathbb{R}_{+} b$ if $\neg\left(a \mathrm{R}_{+} b\right)$

The abbreviation $\mathbb{O}$, in particular, will be of importance in the discussion of boundaries in section 5, where it will be interpreted as an operation of type $\mathcal{P} W \rightarrow \mathcal{P} W$ on the powerset of $W$ through which boundaries can be constructed.

The following lemma introduces the notion of an exact cover of a finite set of events:

LEMMA 1. Let $\mathcal{W}$ be an event structure and let $A$ be a finite multiset of events from $\mathcal{W}$ with enumeration $a_{1}, \ldots, a_{n}$ such that for all $k, a_{k} \mathrm{R}_{+} a_{1}$ and $a_{k} \mathrm{R}_{-} a_{n}$. Then there exists $c$ such that for all $k, a_{k} \preceq c, c \mathrm{R}_{+} a_{1}$ and $c \mathrm{R}_{-} a_{n}$. Such $c$ is called an exact cover of $A$

We also define the notion of a "universal cover", namely, an event which covers all other events in the event structure. The "universal cover" represents the unboundedness of time and will be of great importance to model infinite divisibility in section 8 .

DeFinition 3. Let $\mathcal{W}$ be an event structure and $c \in W$. Then $c$ is a universal cover if for any $a \in W$ it holds that $a \preceq c$.

We now provide a justification of these axioms on the basis of Kant's philosophy, since, as we discussed in the previous sections, the axioms must be justified in terms of the categories and the figurative synthesis. In particular, the problematic axioms from a Kantian perspective are the transitivity axioms, the linearity axioms, the covering axiom and the substitution axiom. Some important technical comments are instead given in section 3.3.

\subsection{Philosophical justification of the axioms.}

3.2.1. The transitivity axioms. Transitivity is a strong principle in our context. In particular, let $a, b, c$ be events of self affection in the description of a space. If $a \mathrm{R}_{-} b, b \mathrm{R}_{-} c$ then in the fleeting present during which the figurative synthesis produces event $b$ event $a$ is reproduced, and in the present during which event $c$ is produced event $b$ is reproduced. In order to be able to conclude that $a \mathrm{R}_{-} c$, the two acts of reproduction must be "composed" so as to be able to reproduce event $a$ when event $c$ is produced. Thus, for transitivity to hold such acts of reproduction must necessarily be able to be composed, since temporal relations between two events can only be established when they are both present before me in one consciousness. The necessity of the possibility of composing such reproductions, however, relies on an objective ground that guarantees it and that extends to all appearances. This objective ground is the category of cause, whose influence on the sensibility determines the objective successions expressed via the relations $\mathrm{R}_{-}, \mathrm{R}_{+}$in the first place; since causality is itself transitive, the latter temporal relations are also transitive.

3.2.2. The linearity axioms. To see how the linearity axioms can be justified in terms of the figurative synthesis, consider a set $E$ of events. In general, the events in $E$ represent acts of self-affection in the description of different spaces, such as lines or circles, which might be constructed "simultaneously"; there is then no guarantee that they arise from the description of a single geometrical space, as in Figure 1. Thus, if we represent descriptions of distinct geometrical spaces as subsets of $E$ we obtain a family $\alpha_{0}, \alpha_{1}, \ldots$ of subsets whose union is $E$. Each set $\alpha_{i}$ contains events that pertain to the 
description of the same spatial manifold in outer sense, e.g. a line or a circle. We also assume that any event belongs to exactly one subset, so that the family of subsets is really a partition of $E$. This is a simplification, since events can in principle belong to various acts of description; an event corresponding to the synthesis of part of the side of a triangle, for instance, belongs to the act of description of the side itself and of the whole triangle. Still, this simplification is harmless for the purpose at hand, and we shall assume it. If we now index every event according to the set it belongs to we obtain pairs of the form $\left(\alpha_{i}, e\right)$ with $\alpha_{i} \subseteq E, e \in \alpha_{i}$.

Now, it is clear that when restricted to any given $\alpha_{i}$ the linearity axioms hold, because if $e, e^{\prime} \in \alpha_{i}$ then $\pi_{s}(e), \pi_{s}\left(e^{\prime}\right)$ are two spaces that are in each other's vicinity and are produced in the same act of description, and hence the events of self-affection are causally related. A similar situation holds in the empirical case: a set of events representing attributes of the same empirical appearance satisfies the linearity axioms because the beginning and end of these events can be simultaneously compared due to their relative closeness. ${ }^{15}$ However, this does not ensure that the linearity axioms hold in general for events that belong to different acts of description that may be very far apart in space and time. If the cognizing subject produces two line segments in different directions starting at different places it is not obvious that events of self-affection belonging to the two line segments must satisfy the excluded middle; they could just be encoded as temporally incomparable, and as lying in different timelines.

The justification for the unrestricted use of the linearity axioms in the axiom system above comes is then grounded on the restriction imposed by the category of community on the figurative synthesis, to the effect that all substances must be in "thoroughgoing interaction" and causal determination. This means, in turn, that between any two events pertaining to different acts of description, or, in the empirical case, between any two attributes pertaining to different substances, there must be causal influence - a sort of action at distance which ensures that events are always comparable with respect to their beginning and end, so that they belong to a single time-line. The one-dimensionality of time then requires the action of the category of community in the form of the linearity axioms.

3.2.3. The covering axiom. The justification of the covering axiom introduces a further important theme, the different philosophical status of universal axioms and existential axioms. The logical form of the axioms considered above was universal; thus, these axioms restrict the class of possible temporal forms of experiences by constraining the possible relations of temporal order among events. The covering axiom, instead, posits additional events which the understanding produces of its own and a priori. In the empirical case, this means that events which need not have been perceived are produced by the understanding itself. In the a priori case of self-affection, which grounds the empirical case, this means that events which need not have been actually described by the subject are produced by the understanding itself. We shall term events that are introduced by existential axioms transcendental, as they are postulated by the understanding a priori.

\footnotetext{
${ }^{15}$ Isaac Barrow seems to make a similar point when he states that "time, abstractly speaking, is the continuance of each thing in its own being" (Barrow (1976), Lectio I.), since Arthur (1995) observes that "since some things continue to exist longer than others, these times are durations with respect to the beings in question, and thus are relative measures"; hence, the order of succession of events is indexed by the substances or beings they are modifications or attributes of.
} 
The justification for the covering axiom stems from the category of substance as the "persistence of the real in time", since as we saw in section 2.6 the representation of the persistence of the spaces synthesized in outer sense depends on the influence of the category of substance on the sensibility, which alone enables the intuition of something as persisting in the first place.

Note that the covering axiom turns the preorder $\preceq$ into a directed preorder. ${ }^{16}$ In the case of a finite set of events $E$, iterated application of the covering axiom implies the existence of an event $w$ with the property that $a \preceq w$ for any $a \in E$. We term $w$ a universal cover, which can be thought of as a representation at the level of a single set of events of the infinite, unbounded time of which all times are part, thus capturing Kant's dictum that "different times are only part of one and the same time" (A31-2/B47). Moreover, it was argued in Achourioti and van Lambalgen (2011), and it will be of importance in section 8, that directedness is closely related to the synthesis of the unity of apperception, as it implies that any two representations are related as parts of an encompassing whole representation. As we saw in section 2.6, that any two events $e, e^{\prime}$ must be part of a larger encompassing event $e^{\prime \prime}$ is a precondition for $e, e^{\prime}$ to be in an objective temporal relation.

3.2.4. The substitution axiom and the arrow of time. The substitution axiom states a duality between $\mathrm{R}_{-}, \mathrm{R}_{+}$that is itself the expression of a perfect symmetry between the "past" and the "future". In particular, given an event structure $\mathcal{W}$, one can obtain a "dual" event structure $o p(\mathcal{W})$ by letting $o p(W)=W$ and letting $\mathrm{R}_{+}^{o p(\mathcal{W})}=\mathrm{R}_{-}^{\mathcal{W}}$, $\mathrm{R}_{-}^{o p(\mathcal{W})}=\mathrm{R}_{+}^{\mathcal{W}}$, and $O^{o p(\mathcal{W})}=O^{\mathcal{W}}$. The dualization operation $o p(\cdot)$, which as we shall see is actually an endofunctor in the category of event structures and event maps, effectively exchanges the orientation of the past and the future; therefore, our axioms do not provide a way to choose one orientation over the other. From a Kantian perspective, however, this is not as problematic as it may seem, since the orientation of the timeline must depend on the subsumption of events under concepts, so that a cause and effect relation between types of events also determines what can precede a given even and what cannot. In other words, from a Kantian perspective the direction of time is determined causally - that is, on the basis of causal laws. The formulation of a formal setting in which all this can be analyzed would require the combination of the formal theory presented in this work with the formalization of Kant's transcendental logic developed in Achourioti and van Lambalgen (2011), but this will not feature here.

3.2.5. The temporal operations. On the basis of Kantian philosophy two interpretations can be given of the operations, one in terms of the synthesis of apprehension and one in terms of the category of causality.

In relation to the synthesis of apprehension we interpret the events introduced by $\oplus, \ominus$ as "potential" binding of other events: given a collection $A=\left\{a_{0}, \ldots, a_{n}\right\}$ of events and an event $e$ in the range of $(\oplus, \ominus), e$ binds $A$ if $A=\{a \in E \mid a \preceq e\}$; i.e., $e$ "encompasses" all and only the events in $A$, and represents their potential binding into a unity. In the a priori case of the description of a line these transcendental events represent actual bindings, since in this case all events are produced a priori by the subject and enjoy only the property of spatio-temporal extension; hence, there are here no constraints on binding sets of events into unities. In the empirical case, in which events may be tenure events of a posteriori qualities by means of which they fall under

${ }^{16}$ Recall that a preorder $(P, \leq)$ is directed if for any $x, y \in P$ there exists $z \in P$ with $x, y \leq z$. 
empirical concepts, there are more constraints on what counts as an objective unity; thus, only some of the potential bindings of events are realized - what Kant calls "the comprehension of the manifold given in accordance with the form of sensibility in an intuitive representation".

The second interpretation of the operations relies on the category of causality. We have seen in section 3.2.2 that the category of community is essential to ensure that the linearity axioms hold, and with them the linearity of the determinations of inner sense produced by the figurative synthesis - the linearity of time. We can then justify the operations $\oplus, \ominus$ in terms of the action of the category of causality as follows: given two events $a, b$ we interpret $a \oplus b$ as "the part of $a$ that can be causally influenced by $b$ ", and $a \ominus b$ as "the part of $a$ that can causally influence $b$ ". The existence of the events in the range of $\oplus, \ominus$ is then justified in terms of the transcendental action of the category of causality.

3.3. Remarks on the axioms. We have already provided ample philosophical commentary on the justification of the axiom system; here we limit ourselves to mostly technical and terminological considerations that will be useful later.

3.3.1. Terminology. The full set of axioms in Definition 1 will be referred to as GT, where "GT" stands for geometry of time, a reference to Kant's insistence on the necessity of an "outer" (geometric) representation of time (B154). When we use the term "event structure", then, we shall in general mean a model of GT, unless specified further. In what follows we shall also work in the subsystem $\mathrm{GT}_{0}$ consisting of GT minus the axioms of group (9) and axiom (8), in which the exact covering Lemma 1 does not hold. We also make use of an intermediate axiom system $\mathrm{GT}_{1}=\mathrm{GT}_{0}+(8)$, which will be important in the treatment of boundaries in section 5 .

3.3.2. The axiom system and geometry. The reader will have undoubtedly drawn some diagrams to clarify the meaning of the axioms and of Lemma 1. Now, if we think of the universal cover $c$ of an event structure, if it exists, as the empty form of time, then the geometric content of the axioms is to construct orthogonal projections from events to $c$, in a way analogous to that illustrated in section 2.6. Euclid's Bk.I, Proposition 12 shows how to construct orthogonal projections, and the theory of parallels (Bk. I, Propositions 27 - 32) shows that projection preserves the primitive relations and operations, as well as quantitative relationships. Although these constructions and proofs are simple, they require Euclid's five postulates (as well as his principles for comparison of magnitudes).

A closer relation to Euclidean geometry can also be obtained by considering the following definitions:

DEFINITION 4. A formula in the signature $\left(\mathrm{R}_{+}, \mathrm{R}_{-}, O ; \preceq ; \oplus, \ominus\right)$ is positive primitive if it is constructed using only $\vee, \bigvee, \wedge, \exists, \perp$.

DEFINITION 5. A formula is geometric or a geometric implication if it is of the form

$$
\forall \bar{x}(\theta(\bar{x}, \bar{y}) \rightarrow \psi(\bar{x}, \bar{y}))
$$

where $\theta$ and $\psi$ are positive primitive.

Note that the logical form of Euclid's problems and theorems in the Elements is that of geometric sentences in which disjunctions in the consequence occur very rarely. Now, our axiom system only consists of geometric sentences, and, if we exclude the 
linearity axioms, only of geometric sentences whose consequent does not contain disjunctions, which highlights how their logical form is close to Euclid's spirit.

3.3.3. Total precedence. The reader who is acquainted with the logical literature on time, events and "periods" (Van Benthem, 2013) might be surprised that we do not have, like (Russell, 1936; Walker, 1947; Thomason, 1984, 1989), a primitive relation $P$ encoding complete precedence. This choice was philosophically justified in section 2.4, but the technical justification for using primitives like $R_{+}, R_{-}$instead of precedence is, as we shall see, that they have a natural topological interpretation.

Nevertheless, we can define total precedence by letting $a P b:=a \mathbb{O} b \wedge a \mathbf{R}_{-} b$. It is straightforward to check that our axioms provide us with all the properties of $P$ that are required for the construction of instants from events proposed by Russell and Walker (Pinosio, 2017, p. 176).

3.3.4. Finite model property. The axioms collected in $\mathrm{GT}_{0}$ are universal; as a consequence, $\mathrm{GT}_{0}$ is in a sense complete with respect to finite models. The same result, however, holds for GT. We can formulate the result precisely if we consider the class of geometric formulas, which were argued to be the formal analogue of Kant's judgments in Achourioti and van Lambalgen (2011).

THEOREM 1. Let $\varphi$ be a geometric implication in the signature of $G T$. Then $G T \models$ $\varphi$ iff $\varphi$ holds on all finite models of GT.

Proof. The direction from left to right is trivial. For the direction from right to left, we prove the contrapositive. Assume $G T \not \forall \varphi(\bar{x}, \bar{y})$, where $\varphi(\bar{x}, \bar{y})$ is of the form $\forall \bar{y}(\theta(\bar{x}, \bar{y}) \rightarrow \psi(\bar{x}, \bar{y}))$ for $\theta(\bar{x}, \bar{y}), \psi(\bar{x}, \bar{y})$ positive primitive formulas. Then for some countable structure $\mathcal{M}$,

$$
\mathcal{M} \models G T+\exists \bar{y}(\theta(\bar{x}, \bar{y}) \wedge \neg \psi(\bar{x}, \bar{y})) .
$$

Thus there must be a tuple $\bar{a}$ of objects of $\mathcal{M}$ such that $\mathcal{M} \models \theta(\bar{a}), \neg \psi(\bar{a})$. Since the tuple $\bar{a}$ is finite, Lemma 1 provides us with an object $c$ which covers every object of $\bar{a}$. We can now define a submodel $\mathcal{M}^{\prime}$ of $\mathcal{M}$ having as objects of the domain the objects in $\bar{a}$, the covering event $c$, and all events which can be obtained from these by closing under the operations $\oplus, \ominus$. It is straightforward to check that $\mathcal{M}^{\prime}$ is a submodel of $\mathcal{M}$ and that the equational theory of $\oplus, \ominus$ ensures that $\operatorname{dom}\left(\mathcal{M}^{\prime}\right)$ is finite. Hence $\mathcal{M}^{\prime}$ is the desired finite model.

Note that the formula expressing the existence of a universal cover

$$
\exists x \forall y(y \preceq x)
$$

is true on all finite models of GT but not on arbitrary models, showing that Theorem 1 above cannot be extended beyond geometric formulas.

3.3.5. Standard models. In the context of GT the relation $O$ has a strong, constructive, interpretation as overlap, which is enforced by the axioms of group (9); $\mathrm{GT}_{1}$ (and $\mathrm{GT}_{0}$ ), on the other hand, allow for a weaker interpretation of $O$ as proximity, the relation of being "infinitesimally close". We can highlight the difference in the allowed interpretations of $O$ in GT and $\mathrm{GT}_{1}$ more precisely by considering set-based models for the axioms. Consider in particular the set of all nonempty open intervals of the unit interval $\mathbb{I}=[0,1]$, equipped with its natural order. These intervals are connected and order-convex. We then have the following: 
LEMMA 2. Define the structure E(I) with domain

$$
\{a \subseteq \mathbb{I} \mid \text { a open, nonempty and order-convex }\}
$$

by letting for any $a, b$ :

(1) $a \mathrm{R}_{+} b$ if $a \subseteq \uparrow b$

(2) $a \mathrm{R}_{-} b$ if $a \subseteq \downarrow b$

(3) $a O b$ if $\operatorname{cl}(a) \cap \operatorname{cl}(b) \neq \emptyset$

(4) $a \oplus b=\uparrow b \cap a$ if $\uparrow b \cap a \neq \emptyset$, otherwise undefined

(5) $a \ominus b=\downarrow b \cap a$ if $\downarrow b \cap a \neq \emptyset$, otherwise undefined

Then $E(\mathbb{I}) \models G T_{1}$ but $E(\mathbb{I}) \mid \forall G T$

If in Lemma 2 we interpreted $O$ differently, however, by letting $a O b$ if $a \cap b \neq \emptyset$, we would have obtained a model of GT. Most importantly, GT has a concrete interpretation in the structure of rational open intervals:

Lemma 3. Let $E_{\omega}(\mathbb{I})$ be the structure having as domain all the nonempty open order-convex subsets of $\mathbb{I} \cap \mathbb{Q}$ and as relations those of Lemma 2 , but let aOb if a $\cap \neq \neq \emptyset$. Then $E_{\omega}(\mathbb{I}) \models G T$.

PROPOSITION 1. Given a finite event structure $\mathcal{W}$, there is a finite submodel $\mathcal{V}$ of $E_{\omega}(\mathbb{I})$ which is isomorphic to $\mathcal{W}$.

Where the notion of isomorphism employed here relies on event maps, which shall be defined in section 6.2

3.3.6. The extensionality axiom. Note that we have not included in the axioms the additional strength provided by the extensionality axiom, which forces $\preceq$ to be a partial order:

$$
a \preceq b \wedge b \preceq a \rightarrow a=b
$$

In the presence of the extensionality axiom we could do without the equational theory for $\oplus, \ominus$ (axioms (9)i-(9)1), since those axioms follow straightforwardly from extensionality; moreover, if we also extended the definition of an event structure with an "empty event" 0 , we could turn $\oplus, \ominus$ into total operations and explicitly define $\mathbf{R}_{+}, \mathbf{R}_{-}, O$ in terms of $\oplus, \ominus$.

One must, however, consider whether the extensionality axiom and the empty event are acceptable from a Kantian perspective, since we do not want to be carried away by the formalism: every axiom must be inspected for transcendental content in agreement with Kant's philosophy for its addition to be justified. As far as the empty event is concerned, for instance, there are good Kantian reason to reject it (Pinosio, 2017). Furthermore, from a mathematical standpoint, it is interesting not to explicitly define $\mathrm{R}_{+}, \mathbf{R}_{-}, O$ in terms of $\oplus, \ominus$ because the weaker axiom systems $\mathrm{GT}_{0}$ and $\mathrm{GT}_{1}$ are interesting in their own right, since it is possible to provide a philosophically interesting construction of instants of time that does not rely on $\oplus, \ominus$ (see section 5).

A few words more should be said with respect to the extensionality axiom. Philosophically, the problem with this axiom is that it implies the identity of events existing at exactly the same times. This is in general unacceptable, since such events might for 
instance occur at different places; indeed, Thomason (Thomason, 1989), Russell (Russell, 1936) and Walker (Walker, 1947) do not have such an axiom. Moreover, the axiom would seem to prevent us from expressing the simultaneity of events, since in its presence there cannot be distinct events that are simultaneous. Note that this fact does relate to Kant's views on the matter, since for Kant simultaneity is not a merely temporal notion, but, as we highlighted in section 2.6, it is rather a spatio-temporal notion, so that in inner sense itself there is not simultaneity ("different times are not simultaneous but successive").

On the other hand, there is a case to be made for the extensionality axiom if we assume that the elements of an event structures are merely the "periods" during which events exist, and not the events themselves, following the distinction in Van Benthem (2013). We have seen in the previous chapters that Kant conceives of an event as the act of drawing a certain space in a certain time, and in this sense an event can be decomposed into a time-component and a space-component; if the elements of an event structure are taken to be the time-components of events, then the extensionality axiom is justified. Given an event structure $\mathcal{W}$, spatiotemporal, as opposed to purely temporal, events might then be represented by a set $E$ and a map $e: E \rightarrow W$ that assigns every event to its time-period. This would allow one to discuss simultaneity of events while still assuming $\preceq$ to be a partial order.

In any case, from a mathematical point of view, for the treatment that follows the presence or absence of the extensionality axiom does not make much difference, since in its absence we could always work with setoids rather than sets. Recall that a setoid is simply a set $A$ equipped with an equivalence relation, a "defined" notion of equality. In our setting, the relevant equivalence relation is $\equiv$, so that given an event structure $\mathcal{W}$ it is of no mathematical import whether we work with $\mathcal{W} / \equiv$, the quotient of $\mathcal{W}$ under $\equiv$ enforcing the extensionality axiom, or whether we treat $\equiv$ as our relevant notion of equality. We shall then for the moment work without the extensionality axiom, but assume it starting from section 8 , since as we shall see it substantially simplifies the discussion there contained.

§4. Topologies on event structures and connectedness. In this section we introduce some notions that will be of importance in the next sections on the construction of the Kantian continuum, and we also discuss the connectedness properties of event structures in relation to Kant's dictum that "parts of time are times" but that "time is not composed out of its parts".

Since in what follows we shall make use of topological notions the reader unfamiliar with topology might wish to consult the first chapters of any textbook on the subject, so as to be able to better follow the upcoming discussions.

4.1. Operations on sets of events. The relations $R_{+}, R_{-}$are reflexive and transitive, that is, preorders. Hence, they lend themselves to the well-known construction of Alexandroff topological spaces from preorders (Alexandroff, 1937). We shall then denote with $\mathcal{A}_{+}, \mathcal{A}_{-}$the Alexandroff topologies generated on an event structure $\mathcal{W}$ by $\mathrm{R}_{+}, \mathrm{R}_{-}$respectively. In other words, $\mathcal{A}_{+}$is just the family of subsets $\left\{U \subseteq W \mid a \in U, b \mathbf{R}_{+} a\right.$ implies $\left.b \in U\right\}$, and similarly for $\mathcal{A}_{-}$; the sets in $\mathcal{A}_{+}, \mathcal{A}_{-}$are 
the open sets of the respective topologies. ${ }^{17}$ For simplicity, we also refer to $\mathcal{A}_{+}$as the future topology and to $\mathcal{A}_{-}$as the past topology, and speak of past-open and future-open sets.

Equipping an event structure $\mathcal{W}$ with the past and future topology turns it into a bitopological space(Kelly, 1963). Furthermore, on the basis of $\mathrm{GT}_{0}$ one can prove, using the linearity axioms, that past-open and future-open sets can be totally ordered:

Lemma 4. For any two future-open sets $A, B: A \subseteq B$ or $B \subseteq A$, and similarly for past-open sets

The relations $O, \mathbb{O}$ also have topological meaning. Let $\mathcal{W}$ be an event structure; we define two relations $O, \mathbb{O} \subseteq \mathcal{P} W \times \mathcal{P} W$ as follows:

(1) $A O B$ if $\exists a \in A \exists b \in B a O b$

(2) $A \mathbb{O} B$ if $\forall a \in A \forall b \in B a \mathbb{O} b$

for $A, B \subseteq W$. The two relations $O, \mathbb{O}$ on sets so defined are a sort of proximity relation and apartness relation on $\mathcal{P} W$, respectively. In particular, although $\mathbb{O}$ is a symmetric relation, it gives rise to two asymmetric operations on open sets, where the asymmetry derives from the existence of the past and future topologies:

Definition 6. Let $\mathcal{W}$ be an event structure. The unary operations $(\cdot) \mathbb{O}: \mathcal{A}_{+} \rightarrow$ $\mathcal{P} W$ and $\mathbb{O}(\cdot): \mathcal{A}_{-} \rightarrow \mathcal{P} W$ are defined by letting:

$$
A \mathbb{O}=\mathbb{O} A=\{a \in W \mid \forall b \in A a \mathbb{O} b\}
$$

for $A \in \mathcal{A}_{+}$future-open or $A \in \mathcal{A}_{-}$past-open.

LEMMA 5. Let $\mathcal{W}$ be an event structure and let $U, V$ be past-open and future-open respectively. The following hold:

1. UO is future-open.

2. $\mathbb{O} V$ is past-open.

Thus, the relation $(1)$ can be used to define two unary operations, one from the set $\mathcal{A}_{-}$of past-open sets of an event structure $\mathcal{W}$ to the set $\mathcal{A}_{+}$of future-open sets, and the other from the set of future-open sets to the set of past-open sets. Note however that, even though $\mathcal{A}_{+}, \mathcal{A}_{-}$are complete distributive lattices (complete Heyting algebras), the two operations are not lattice maps, as they do not preserve meets. These operations will prove useful to provide a definition of Kantian boundary in the next section.

Finally, recall that the defined covering relation $\preceq$ is transitive and reflexive, hence a preorder; it then also gives rise to an Alexandroff topological space. In the sequel we shall make use of the self-duality of the Alexandroff topologies generated by $\preceq$, and use both the topology whose closed sets are the upsets and the topology whose closed sets are the downsets. It will always be clear from the context which topology we are using.

\footnotetext{
${ }^{17}$ Note that $\mathcal{A}_{+}$is closed under arbitrary unions and intersections. Indeed, a topology on a set $X$ is just a family $\tau \subseteq \mathcal{P} X$ of subsets of $X$ which (i) is closed under arbitrary unions and intersections and (ii) contains $X$ and $\emptyset$; an Alexandroff topology is additionally closed under arbitrary intersections.
} 
4.2. Connectedness of event structures. Continuity and connectedness as synthetic a priori principles for time occur under various guises in Kant's works. As far as connectedness of time is concerned, in particular, one finds two common characterizations of this notion. The first is mereological in nature, stating that "parts of time" are themselves times that cannot be "detached" from the encompassing whole of time, i.e., parts of time "can be distinguished, but not separated" (R.4425, 17:541), so that divisio non est realis, sed logica. The second characterization has to do with the instants in time, and states that there are no "jumps" or "leaps" from one state of a substance to another, without intermediate transitions in between. ${ }^{18}$ In this section we analyze briefly the first notion of connectedness in relation to event structures, while in the following sections on boundaries in time we shall mostly focus on the second characterization.

In the context of point-set topology, connectedness is defined as an indecomposability condition: a topological space is connected if there are no disjoint non-empty open sets whose union is the whole space. Kant's notion of indecomposability is much stronger, but before we can elucidate it we must define what it means for a space to be connected in a bitopological setting. Since the future-open sets are linearly ordered by inclusion (Lemma 4), any event structure is trivially connected in the $R_{+}$topology, and likewise for the $R_{-}$topology. To be able to say something more interesting we therefore need both topologies:

DEFINITION 7. The event structure $\mathcal{W}$ is biconnected if there are no non-empty $U, V$ such that $U$ is past-open, $V$ is fut-open, $U \cap V=\emptyset$ and $U \cup V=W$.

As a consequence of the covering axiom, we obtain the following:

Lemma 6. Let $\mathcal{W}$ be an event structure. Then $\mathcal{W}$ is biconnected.

The covering axiom implies a still stronger form of connectedness, however, which can be better seen by considering the join topology on an event structure $\mathcal{W}$, that is, the topology $\mathcal{A}_{+} \vee \mathcal{A}_{-}$on $\mathcal{W}$ generated by taking $\mathcal{A}_{+} \cup \mathcal{A}_{-}$as a subbase. It is straightforward to see that in this topology open sets are downsets with respect to $\preceq$; we could then say that in this topology open sets are closed under the relation "is a logical part of". This in turn implies that closed sets are upsets with respect to $\preceq$, as the complement of a downset is an upset. This immediately yields:

LEMMA 7. Let $\mathcal{W}$ be an event structure and let $A, B \subseteq W$ be non-empty sets closed in $\mathcal{A}_{\mathrm{R}_{+}} \vee \mathcal{A}_{\mathrm{R}_{-}}$. Then $A \cap B$ is not empty

The result above suggests that the following stronger notion of connectedness is more appropriate for event structures:

DEFINITION 8. A topological space is ultra-connected if any two non-empty closed sets have non-empty intersection.

Note that in our bitopological setting, this concept is non-trivial only for sets closed in $\mathcal{A}_{\mathrm{R}_{+}} \vee \mathcal{A}_{\mathrm{R}_{-}}$, hence in the preceding definition "closed" will be taken in this sense. We then have:

${ }^{18}$ Thus Kant:

There is nothing simple in appearance, hence no immediate transition from one determinate state (not of its boundary) into another [ ... ] a hiatus, a cleft, is a lack of interconnection among appearances, where their transition is missing. (R.4756, 17:699) 
LEMMA 8. Event structures with the $\mathcal{A}_{\mathrm{R}_{+}} \vee \mathcal{A}_{\mathrm{R}_{-}}$topology are ultra-connected.

In the context of finite event structures the above lemma reduces to the statement that there exists a universal cover.

We can also use the ultra-connectedness of event structures to model Kant's dictum that time cannot be "made up" from its parts. Indeed, note that given an event structure $\mathcal{W}$ and an event $a \in W$, the principal ideal $\downarrow \preceq a$ of $a$ under $\preceq$ is open in $\mathcal{A}_{\mathrm{R}_{+}} \vee \mathcal{A}_{\mathrm{R}_{-}}$, and it is easy to show that $\downarrow \preceq a$ is itself an event structure. We might then take these ideals to denote "parts of time":

DEFINITION 9. Let $\mathcal{W}$ be an event structure and let $a \in W$. Then $\downarrow_{a} \preceq$, the principal ideal generated by $a$ in the $\preceq$ ordering, is a part of time in $\mathcal{W}$. A part of time $\mathcal{W}^{\prime}$ is said to be proper if $\mathcal{W}^{\prime} \neq \mathcal{W}$.

Now, it is straightforward to see that ultra-connectedness can be formulated for open sets of $\mathcal{A}_{\mathrm{R}_{+}} \vee \mathcal{A}_{\mathrm{R}_{-}}$as follows: for any open sets $U, V, W=U \cup V$ implies either $W=U$ or $\mathcal{W}=V$. This immediately yields:

Proposition 2. Lete $\mathcal{W}$ be an event structure. Then $W$ cannot be written as the union of proper parts of time.

The above proposition goes a long way to capture Kant's idea that time "as a whole" cannot be composed or constructed from its parts. It now remains to investigate the definition of connectedness as absence of "clefts" in time, for which we must turn to the discussion of instants or boundaries of time.

§5. Boundaries as limitations. The main aim of this section is to discuss the construction of Kantian boundaries and show that the set of events can be given the structure of a one dimensional continuum. We argue as follows. Kant conceives of boundaries in time as "limitations"; that is, for Kant time is prior to its parts, which have the same structure as time itself - in particular there are no smallest parts allowing the construction of time as a set:

Space therefore consists only of spaces, time of times. Points and instants are only boundaries, i.e., mere places of their limitation; but places always presuppose those intuitions that limit or determine them, and from mere places, as components that could be given prior to space or time, neither space nor time can be composed. (CPR A170/B212)

This notion of boundary, however, is a topological concept, not an order-theoretic one. Informally, a temporal boundary in an event structure $\mathcal{W}$ determines a set of events $P \subseteq W$ in the past of that boundary, and likewise a set of events $F \subseteq W$ in the future of the boundary, so that it "limits" but simultaneously "links" the past and the future, as not only Kant, but also Aristotle, held (see Passage (3) in section 2.3). Furthermore, $P$ and $F$ ought to be $\mathbb{O}$-apart; this implies that they are set-theoretically disjoint, and the complement of $P \cup F$ can be viewed as a representation of the temporal boundary between $P$ and $F$, which we might rightly call the present $C$. The topologies defined in the previous section will then have a temporal meaning, as the open sets of $\mathcal{A}_{+}$can be used to represent the future of a boundary, the open sets of $\mathcal{A}_{-}$its past and the open sets of $\mathcal{A}_{\preceq}$ its present. Note moreover that the division into past and future of a boundary is relative to the domain $W$ of $\mathcal{W}$, and all $e \in W$ may be situated in the past from the standpoint of the now; that is, if $a \in F$ it does not mean that $a$ is still to come. 
Temporal progression in the sense of coming to be is represented not by a single event structure, but by a system of event structures linked by continuous maps, as we shall see in section 8 .

5.1. Boundaries from closure operators. We shall provide a better definition of Kantian boundaries by means of a construction starting from an even structure $\mathcal{W}$ and the set of pairs of the form $(U, V)$ with $U \subseteq W$ past-open and $V \subseteq U$ future-open. This construction really amounts to defining two closure operators, one on $\mathcal{A}_{+}$and the other on $\mathcal{A}_{-}$. A boundary will then be represented as a pair $(P, F)$ where $P, F$ are closed with respect to the former and the latter closure operator, respectively. Note, most importantly, that these closure operators are distinct from the topological closure operators of the past and future topologies themselves, so that the reader should not confuse the two.

DEFINITION 10. Let $\mathcal{W}$ be an event structure and define endomaps $L(\cdot), R(\cdot)$ on the set of past-open and future-open sets, respectively, by letting

$$
\begin{aligned}
L(\cdot): U \mapsto \mathbb{O}(U \mathbb{O}) \\
R(\cdot): V \mapsto(\mathbb{O} V) \mathbb{O}
\end{aligned}
$$

Where $U, V \subseteq W$ are respectively past-open and future-open.

We then have:

THEOREM 2. Let $\mathcal{W}$ be an event structure and let $L(\cdot), R(\cdot)$ be as in Definition 10. Then the following hold:

(1) $L(\cdot)$ is monotone: $U \subseteq U^{\prime}$ entails $\mathbb{O}(U \mathbb{O}) \subseteq \mathbb{O}\left(U^{\prime} \mathbb{O}\right)$

(2) $L(\cdot)$ is extensive: $U \subseteq \mathbb{O}(U \mathbb{O})$

(3) $R(\cdot)$ is monotone and extensive

(4) for any $U$ we have $L(U)=L L(U)$, and similarly $R(V)=R R(V)$

(5) if $U$ is $L(\cdot)$-closed, then $V=U \mathbb{O}$ is $R(\cdot)$-closed and we have $\mathbb{O} V=U$. Analogously if $V$ is $R(\cdot)$-closed, then $U=\mathbb{O} V$ is $L(\cdot)$-closed and $U \mathbb{O}=V$

(6) the set of $L(\cdot)$-closed sets (resp. $R(\cdot)$-closed sets) is a complete linear order under inclusion

(7) $W$, $\emptyset$ are closed for both operators.

ProOF. For claim (1) one checks that $U \subseteq U^{\prime}$ entails $U^{\prime} \mathbb{O} \subseteq U \mathbb{O}$, which in turn entails $\mathbb{O}(U \mathbb{O}) \subseteq \mathbb{O}\left(U^{\prime} \mathbb{O}\right)$; these latter sets are past-open by Lemma 5 .

For claim (2) we note that Lemma 5 implies $\mathbb{O}(U \mathbb{O})$ is past-open. Choose $a \in U, b \in$ $U \mathbb{O}$, then by definition $a \mathbb{O} b$, hence $a \in \mathbb{O}(U \mathbb{O})$. Claim (3) is proven similarly.

For claim (4), observe that by claim (1) we have

$$
\mathbb{O}(U \mathbb{O}) \subseteq \mathbb{O}((\mathbb{O}(U \mathbb{O})) \mathbb{O})
$$

and by claim (3), setting $V=U \mathbb{O}$, we obtain the converse inclusion, whence

$$
\mathbb{O}(U \mathbb{O})=\mathbb{O}((\mathbb{O}(U \mathbb{O})) \mathbb{O})
$$

To prove claim (5) we note that $U=\mathbb{O}(U \mathbb{O})$ implies $(\mathbb{O} V) \mathbb{O}=(\mathbb{O}(U \mathbb{O})) \mathbb{O}=$ $U \mathbb{O}=V$. 
To prove claim (6), let $U=\bigcup_{i} U_{i}$ be a union of $L(\cdot)$-closed sets. Then $U$ need not be $L(\cdot)$-closed, but $\mathbb{O}\left(\bigcup_{i} U_{i} \mathbb{O}\right)$ is the least closed set larger than the $U_{i}$. Furthermore, given a pair $(U, V)$ we can construct two increasing sequences, where $\subseteq$ is interpreted coordinate-wise:

$$
(U, V) \subseteq(U, U \mathbb{O}) \subseteq(\mathbb{O}(U \mathbb{O}), U \mathbb{O})
$$

and

$$
(U, V) \subseteq(\mathbb{O} V, V) \subseteq(\mathbb{O} V,(\mathbb{O} V) \mathbb{O})
$$

Since the past-opens are linearly ordered by $\subseteq$ (Lemma 4), we may fuse the two sequences by ordering them linearly according to the first coordinate

$$
(U, V) \leq(\mathbb{O}(U \mathbb{O}), V) \leq((\mathbb{O} V, V) \leq(\mathbb{O} V,(\mathbb{O} V) \mathbb{O})
$$

which gives, by Theorem 2 (5), for any past-open $U$ least and greatest extensions that are fixpoints.

The proof of claim (7) is straightforward.

The theorem above shows that $L(\cdot), R(\cdot)$ are closure operators on the lattices of pastopen and future-open sets of an event structure, respectively. We can now define boundaries of an event structure in terms of these closure operators by considering $L(\cdot), R(\cdot)$ as a single operation $(L(\cdot), R(\cdot))$ on the product lattice $\mathcal{A}_{-} \times \mathcal{A}_{+}$of past-open and future-open sets, as follows:

Definition 11. Let $\mathcal{W}$ be an event structure. A boundary is a tuple $(P, C, F)$ of subsets of $W$ such that the following hold:

1. $P, F$ are past-open and future-open respectively

2. $(P, F)$ is closed for the closure operator $(L(\cdot), R(\cdot))$

3. $F=P \mathbb{O}$ (and hence $P=\mathbb{O} F$ because of Theorem 2 (5))

4. $C=(P \cup F)^{c}$

LEMMA 9. Let $\mathcal{W}$ be an event structure and let $a, b \in W$ be such that $a \mathbb{O} b, a \mathbf{R}_{-} b$, i.e., a completely precedes $b$. Then there exists a boundary $(P, C, F)$ with $a \in P, b \in F$.

The boundaries from Definition 11 satisfy various properties, which allow us to provide an explicit definition of when a triple of sets is such a boundary. In particular:

PROposition 3. Let $\mathcal{W}$ be an event structure and let $(P, C, F)$ be a boundary according to Definition 11. Then the following hold:

(1) $P$ and $F$ are $\mathbb{O}$-apart

(2) $P \cup C \cup F=W$ and $P, C, F$ are all disjoint.

(3) $C$ is empty if and only if either $P$ or $F$ are empty

(4) $C \neq W$

(5) For any $a \in W$, if a overlaps with an event in $P$ and an event in $F$ then it belongs to $C$

(6) For any $a, b \in C a O b$

(7) For any $a \in C$ if $P$ is not empty then there is $b \in P$ with $a O b$

(8) For any $a \in C$ if $F$ is not empty then there is $b \in F$ with $a O b$ 
In light of Proposition 3 we then have the following characterization of boundaries:

Proposition 4. Let $\mathcal{W}$ be an event structure. A tuple $(P, C, F)$ of subsets of $W$ is a boundary if and only if it satisfies:

(1) $P \cup C \cup F=W$

(2) If one of $P$ or $F$ are empty then so is $C$

(3) $P$ is past-open and $F$ is future-open

(4) $P$ and $F$ are $\mathbb{O}$-apart

(5) For any $a \in C$ there is $b \in P$ with $a O b$

(6) For any $a \in C$ there is $b \in F$ with $a O b$

We can now show that the set of boundaries defined from an event structure $\mathcal{W}$ can be linearly ordered, as follows:

PROPOSITION 5. Let $\mathcal{W}$ be an event structure and $\leq$ be a binary relation on the set of boundaries of $\mathcal{W}$ defined by letting $(P, C, F) \leq\left(P^{\prime}, C^{\prime}, F^{\prime}\right)$ if $P \subseteq P^{\prime}$. Then $\leq$ is a complete linear order.

PROOF. The inclusion order is linear because pasts of boundaries are past-open sets and Lemma 4. The linear order is complete because of Theorem 2 (6).

COROLLARY 1. The set of boundaries of an event structure equipped with the order topology is Hausdorff and compact.

PRoOF. The first observation is standard. Compactness follows from the fact that in a linear order that is a complete lattice, each closed interval is compact, combined with Proposition 5.

DEFINITION 12. Let $\mathcal{W}$ be an event structure. The space of boundaries $B(\mathcal{W})$ of $\mathcal{W}$ is the set of boundaries according to Definition 11 ordered under inclusion of pasts as in Proposition 5.

In what follows, we shall often write $x \in B(\mathcal{W})$ for a given boundary of $\mathcal{W}$, and denote the past, present and future of $x$ as $P_{i}, C_{i}, F_{i}$, respectively. Note that $B(\mathcal{W})$ can be considered as a linearly ordered topological space by taking the order topology.

The following result, which we shall need in the sequel, only holds if the event structure is a model of GT:

LEMMA 10. Let $\mathcal{W}$ be an event structure that satisfies the full set of axioms GT and let $x, y \in B(\mathcal{W})$ be boundaries such that $x<y$. Then there exists an event $a \in \mathcal{W}$ such that $a \in P_{y} \cap F_{x}$.

PRoOF. Since $x<y$ we must have that $P_{x} \subset P_{y}$ hence there exists $a \in P_{y}, a \notin P_{x}$. If $a \in F_{x}$ we are done. Otherwise $a \notin F_{x}, a \in C_{x}$. By Proposition 4 since $C_{x}$ is not empty then $F_{x}$ is also not empty and there exists $c \in F_{x}$ with $a O c$. We let $b=a \oplus c$ and we are done.

5.2. Boundaries and the infinity of time. The boundaries introduced in the previous section capture Kant's conception of boundaries in time as "limitations" between parts of time quite well. There is an interesting philosophical distinction to be made, however, between what we shall term "two-sided boundaries" and "formal boundaries".

DEFINITION 13. Let $\mathcal{W}$ be an event structure and $x \in B(\mathcal{W})$ a boundary. Then $x$ is $a$ two-sided boundary if $C_{x}$, and consequently $P_{x}, F_{x}$ are not empty, while it is a formal boundary if $C_{x}$ is empty. 
There are only two formal boundaries, those of the form $(\emptyset, \emptyset, W),(W, \emptyset, \emptyset)$, which are respectively the minimum and maximum of the complete lattice of boundaries. Formal boundaries have a different intepretation than two-sided boundaries, ${ }^{19}$ since they lie strictly "beyond" any event, and can be taken as a formal representation at the level of boundaries of Kant's construal of the infinity of time.

More precisely, let us recall Kant's notion of infinity of time:

The infinitude of time signifies nothing more than that every determinate magnitude of time is only possible through limitations of a single time grounding it. The original representation of time must there be given as unlimited. But where the parts themselves [...] can be determinately represented only through limitation, there the entire representation cannot be given through concepts [...] but immediate intuition must ground them. (A31-2/B47-8)

We interpret "magnitude of time" as a function defined on intervals that are determined by boundaries. Given Kant's concept of number, a "determinate magnitude" is a function that takes only rational values; since magnitudes must be continuous, and the values of the "determinate magnitude" are closed points, the domain of the magnitude must consist of closed intervals. In an event structure $\mathcal{W}$, the time elapsed until now corresponds to a closed interval, with now represented as the maximum of the set of two-sided boundaries; that is, $N=\bigvee\{x \in B(\mathcal{W}) \mid x$ is two-sided. $\}$. Obviously time does not stop now; indeed, the events contained in $F_{N}$, which are contained in the interval between $N$ and the formal boundary $(W, \emptyset, \emptyset)$ and, as it is easy to see, are pairwise overlapping, represent the potentiality for the coming to be of the future. In Husserlian terms, they are a formal representation of the protension of the subject towards the future. Thus, the interval between the maximum and minimum of the set of two-sided boundaries - the now, as we have seen, and what we might call the origin is the "bounded" time; while the whole space $\mathcal{W}$, which is contained between the two formal boundaries, is the "infinite" or "unbounded" time.

§6. Infinitesimal intervals and the general form of Kantian continua. The construction of boundaries presented in the previous section is quite satisfactory, not only mathematically but also philosophically, as it captures closely Kant's (and Aristotle's) claims about boundaries in time. It is, however, wanting from one respect: the space of boundaries $B(\mathcal{W})$ of an event structure $\mathcal{W}$ is compact but it is not necessarily connected, and hence it can hardly be said to be a continuum. The problem lies in the fact that a total order is connected only if it does not have any jumps, i.e., no pair of points $(x, y)$ with $x<y$ and $\neg \exists z x<z, z<y$. However, we cannot certainly impose an axiom on event structures that ensures density of boundaries, as (Russell, 1936) or (Walker, 1947) would have it, because this would rule out finite models for our axioms, and we are especially interested in these finite models as a correlate to the figurative synthesis.

${ }^{19}$ Note that Aristotle only recognizes two-sided boundaries; he argues that instants, since they are limitations of parts of time, cannot have empty past or future, but are always preceded by a time:

Now since [. . . ] the moment [is] a kind of middle-point, uniting as it does in itself [. . . ] a beginning of future time and an end of past time, it follows that [...] there must always be time on both sides of it (Physics, 251b 13-17)

Clearly, two-sided boundaries capture exactly Aristotle's boundaries. 
We shall then pursue a different strategy and fill the jumps in the set of boundaries with a new sort of "instants" that we shall rather call "infinitesimal intervals", since they represent the fleeting time that exists between any two boundaries. These infinitesimal intervals act as a sort of "glue" between boundaries, in the sense of Brouwer's continuist conception of boundaries:

[The ur-intuition is] the substratum of all perception of change, which is divested of all quality, a unity of continuous and discrete, a possibility of the thinking together of several units, connected by a "between", which never exhausts itself by the interpolation of new units. $^{20}$

Moreover, the infinitesimal intervals are closely related to Kant's notion of infinitesimals generated by a flowing magnitude, which will be considered in 10 . Hence, we adopt a terminology that is slightly more specialized than Kant's: the term instant will refer to either boundaries or infinitesimal intervals in time. In this section we again assume only $\mathrm{GT}_{0}$, unless specified further.

6.1. Infinitesimal intervals. We begin with the important definition of a maximal overlapping class of events, already employed in Russell (1936):

Definition 14. Let $\mathcal{W}$ be an event structure and $A \subseteq W$. We say that $A$ is an overlapping class of events if any two events in the class overlap. It is a maximal overlapping class if it is an overlapping class such that there is no overlapping class $B$ with $A \subset B$.

We can now relax Definition 11 to the definition of an instant:

Definition 15. Let $\mathcal{W}$ be an event structure. An instant of $\mathcal{W}$ is a tuple $(P, C, F)$ of subsets of $W$ such that the following hold:

(1) $P, F$ are past-open and future-open respectively

(2) $(P, F)$ is closed for the closure operator $(L(\cdot), R(\cdot))$

(3) $F \subseteq P \mathbb{O}$ (hence $P \subseteq \mathbb{O} F$ because of Theorem 2)

(4) $C=(P \cup F)^{c}$ is an overlapping class

The point of the definition of an instant is to relax Definition 11 so that the pair $P$ and $F$ need not necessarily be "adjacent" or "touching" but can be apart, as long as the cleft between them is "small enough" that no determination of succession, in the form of complete precedence, is possible within it. Indeed, note that condition (3) is now just equivalent to requiring $P, F$ to be $\mathbb{O}$-apart. Thus, while in Definition $11 P$ and $F$ are always "touching", here we aim to allow them to be "almost touching". In this latter case the cleft between them, i.e. the present $(P \cup F)^{c}$, will be interpreted as an "enduring" present, by means of which we shall elucidate further Kant's notion of the description of a space (see section 10). We then posit the following:

Definition 16. Let $\mathcal{W}$ be an event structure and let $(P, C, F)$ be an instant. Then $i$ is an infinitesimal interval if $F \subset P \mathbb{O}$, i.e., $F$ is a strict subset of $P \mathbb{O}$.

Note that if an instant is not an infinitesimal interval then it is simply a boundary in agreement with Definition 11, since condition (4) of Definition 15 follows from Definition 11 (see Proposition 3 (6)). Hence the properties that we proved in the previous

${ }^{20}$ For the source of this quote and an illuminating discussion of Brouwer's continuum see (van Dalen, 2009). 
section still hold conditionally for these boundaries. For the infinitesimal intervals we instead have:

PROPOSITION 6. Let $\mathcal{W}$ be an event structure and let $i$ be an infinitesimal interval according to Definition 15. Then the following hold:

(1) Properties (1), (2), (5) and (6) of Proposition 3 hold of $i$

(2) $C_{i}$ is a maximal overlapping class

PROOF. We only show that $C_{i}$ is a maximal overlapping class as the proof for the other properties is merely a variation on the proof of Proposition 3. First note that if $i$ is an infinitesimal interval then $F_{i} \subset P_{i} \mathbb{O}$ and $P_{i} \subset \mathbb{O} F_{i}$. Let then $a \in W$ be such that $a O b$ for any $b \in C_{i}$. We show that $b \in C_{i}$, i.e. $b \notin P_{i}, b \notin F_{i}$. Assume towards a contradiction that $b \in P_{i}$. Since $F_{i} \subset P_{i} \mathbb{O}$ there exists $c \in W$ with $c \in P_{i} \mathbb{O}$ and $c \notin F_{i}$; it then follows from property (2) of Proposition 3 that $c \in C_{i}$, and by assumption that $c O b$, which yields a contradiction. The proof for $b \notin F_{b}$ is analogous, and we are done.

Note that it is not the case that if $C_{i}$ is a maximal overlapping class then $i$ is an infinitesimal interval, unless one imposes additional conditions on event structures.

As we already did in section 5 , of course, we can explicitly characterize when a tuple of the form $(P, C, F)$ is an instant:

Proposition 7. Let $\mathcal{W}$ be an event structure. A tuple $(P, C, F)$ of subsets of $W$ is an instant if and only if it satisfies:

(1) $P \cup C \cup F=W$

(2) $P, F$ are disjoint from $C$

(3) $P$ is past-open and $F$ is future-open

(4) $P$ and $F$ are $\mathbb{O}$-apart

(5) $C$ is an overlapping class

(6) For any $a \in C$ there is $b$ such that $a O b$ and $b \mathbb{O} c$ for any $c \in P$

(7) For any $a \in C$ there is $b$ such that $a O b$ and $b \mathbb{O} c$ for any $c \in F$

Of course, the question now arises whether we can endow the set of instants with a total order and a topology that improves on what was achieved in the previous section. To this effect, recall that a jump of a linear $\operatorname{order}(L, \leq)$ is a pair of elements $x, y \in L$ such that $x<y$ and there exists no $z \in L$ with $x<z<y$. The following result shows that if we construct the linear order of boundaries $B(\mathcal{W})$ on an event structure, the jumps of the linear order are in one to one correspondence with the infinitesimal intervals as introduced above:

LEMMA 11. Let $\mathcal{W}$ be an event structure and let $B(\mathcal{W})$ be its set of boundaries. Then there exists a bijection $j$ from the set of jumps of $B(\mathcal{W})$ to the set of infinitesimal intervals on $\mathcal{W}$.

Proof. Let $\mathcal{W}$ be an event structure. We construct a bijection between the set of jumps of $B(\mathcal{W})$ and the set of infinitesimal intervals of $\mathcal{W}$ as follows. Let $x, y \in B(\mathcal{W})$ such that $(x, y)$ is a jump, and consider the tuple $\left(P_{x}, C, F_{y}\right)$, where $C=\left(P_{x} \cup F_{y}\right)^{c}$; we claim that this tuple is an infinitesimal interval. Conditions (1), (2), (3) of Definition 15 are trivially verified; we show that $C$ is an overlapping class. Indeed, choose $a, b \in$ $\left(P_{x} \cup F_{y}\right)^{c}$ and assume towards a contradiction that $a \mathbb{O} b$. Then because of the linearity axioms one of $a, b$ precedes the other, say $a$ precedes $b$. But then by Lemma 9 there 
exists a boundary $z$ with $a \in P_{z}, b \in F_{z}$, and it is a straightforward matter to check that $P_{x} \subset P_{z} \subset P_{y}$ so that $x<z<y$; this leads to a contradiction since $(x, y)$ was assumed to be a jump, and we are done. To show that $\left(P_{x}, C, F_{y}\right)$ is an infinitesimal interval it remains to show that $P_{x} \mathbb{O} \neq F_{y}$, but this follows because otherwise $x=y$, but $(x, y)$ was supposed to be a jump.

We can thus define a map $j$ from the set of jumps of $B(\mathcal{W})$ to the set of infinitesimal intervals by associating to any jump $(x, y)$ the infinitesimal interval $\left(P_{x}, C, F_{y}\right)$ defined as above. Clearly, if $(x, y),(z, w)$ are two distinct jumps then $j(x, y) \neq j(z, w)$ since they will have distinct pasts, hence the map is injective. To see that $j$ is surjective let $i$ be an infinitesimal interval and consider $x=\left(P_{i},\left(P_{i} \cup P_{i} \mathbb{O}\right)^{c}, P_{i} \mathbb{O}\right)$ and $y=$ $\left(\mathbb{O} F_{i},\left(\mathbb{O} F_{i} \cup F_{i}\right)^{c}, F_{i}\right)$; clearly $x, y \in B(\mathcal{W})$ with $x<y$ since $P_{i} \subset \mathbb{O} F_{i}$ as $i$ is an infinitesimal interval. We now claim that $(x, y)$ is a jump. Indeed, assume towards a contradiction that there exists $z \in B(\mathcal{W})$ with $x<z<y$. Hence $P_{i} \subset P_{z} \subset \mathbb{O} F_{i}$ which implies $F_{z} \subset F_{i}$. Hence there are $a \in P_{z}, a \notin P_{i}$ and $b \in F_{z}, b \notin F_{i}$, hence $a, b \in C_{i}$ and $a \mathbb{O} b$, but $C_{i}$ is an overlapping class, contradiction.

Note that the results above allow us to linearly order the set of infinitesimal intervals on an event structure $\mathcal{W}$, where this linear order is simply that induced by the linear order of the jumps of $B(\mathcal{W})$. Hence, we obtain:

LEMMA 12. Let $\mathcal{W}$ be an event structure and let $i$ be an infinitesimal interval, then there are $x, y \in B(\mathcal{W})$ with $x=i^{-}, y=i^{+}$.

PROPOSITION 8. Let $\mathcal{W}$ be an event structure. The set of infinitesimal intervals of $\mathcal{W}$, denoted $I(\mathcal{W})$, is linearly ordered by letting $i \leq i^{\prime}$ if $P_{i} \subseteq P_{i^{\prime}}$ for any $i, i^{\prime} \in I(\mathcal{W})$.

Most importantly the whole set of instants on $\mathcal{W}$ according to Definition 15 - the boundaries and infinitesimal intervals - can be linearly ordered. We must, however, define the order by requiring not only inclusion of pasts but also inclusion of futures, because in general a boundary and infinitesimal interval can have the same past or the same future, but not both. In particular, the past and future of an infinitesimal interval $i \in I(\mathcal{W})$ are determined by the past and future of the boundaries $x, y \in B(\mathcal{W})$ such that $j(i)=(x, y)$, where $j$ is the bijection of Lemma 11. We then have:

PROPOSITION 9. Let $\mathcal{W}$ be an event structure. The set of instants of $\mathcal{W}$ according to Definition 15, denoted with $K(\mathcal{W})$, is totally ordered by letting $x \leq y$ if $P_{x} \subseteq P_{y}$ and $F_{y} \subseteq F_{x}$ for any $x, y \in K(\mathcal{W})$, and we have:

(1) $\leq$ is a complete linearly ordered lattice

(2) $B(\mathcal{W})$ is dense in $I(\mathcal{W})$, that is for any $i, i^{\prime} \in I(\mathcal{W})$ with $i<i^{\prime}$ there exists $x \in B(\mathcal{W})$ with $i<x<i^{\prime}$

(3) Two boundaries $x, y \in B(\mathcal{W})$ define a jump $(x, y)$ if and only if there exists exactly one infinitesimal interval $i \in I(\mathcal{W})$ with $x<i<y$.

We must now address the question of which topology to impose on $K(\mathcal{W})$, which is essential to discuss Kant's properties of time that have to do with continuity and connectedness. We have seen that the order topology of $B(\mathcal{W})$ can be totally disconnected, for instance when $B(\mathcal{W})$ is finite. In order to remedy this situation, we introduced the infinitesimal intervals; Lemma 11 shows that $I(\mathcal{W})$ acts as a kind of "filling glue" for jumps in $B(\mathcal{W})$. We cannot, however, impose the order topology on $K(\mathcal{W})$, because this would just reproduce the previous situation and yield in general a disconnected 
topological space, since an infinitesimal interval and one of the adjacent boundaries would induce a partition of the space into disjoint open sets. We shall then impose on $K(\mathcal{W})$ the order topology of $B(\mathcal{W})$ rather than the order topology of $K(\mathcal{W})$ itself; in other words, the topology on $K(\mathcal{W})$ will be generated only by the subbasis of orderopen rays of $B(\mathcal{W})$, as follows:

THEOREM 3. Let $\mathcal{W}$ be an event structure. Let $\tau \subseteq \mathcal{P} K(\mathcal{W})$ be the topology on $K(\mathcal{W})$ generated by the subbase of sets of the form $\{(x, \leftarrow) \mid x \in B(\mathcal{W})\} \cup\{(x, \rightarrow) \mid$ $x \in B(\mathcal{W})\}$. Then $(K(\mathcal{W}), \tau)$ is a compact connected $T_{0}$ ordered topological space such that every boundary is closed and every infinitesimal interval is open.

We shall abuse our notation and indicate with $K(\mathcal{W})$ the ordered topological space of Theorem 3. From the perspective of ordered topological spaces, Theorem 3 shows that $K(\mathcal{W})$ is a COTS, that is, a connected ordered space (Khalimsky, Kopperman, $\&$ Meyer, 1990). This fact highlights that there is a close relation between the theory of well-formed ordered spaces (of which COTS are a special case), as studied in Kopperman, Kronheimer, and Wilson (1998), and event structures as studied in this paper (Pinosio, 2017).

We now have a way to construct a continuum from events which closely captures not only Kant's insights on the matter, but also the general continuist insight expressed by Brouwer in Passage (7) that between any two instants of time there is an inexhaustible "in-between": the infinitesimal intervals capture exactly the latter, and their role in this sense will become clearer once we consider the potential infinite divisibility of time in section 8 .

6.2. Representation of events as intervals. A fundamental question in the logical literature on the construction of time from events is: given an event structure, how can an event be represented as an interval of its space of instants so that the relevant temporal relations are preserved? In our setting, this amounts to representing an event in an event structure $\mathcal{W}$ as an interval of $K(\mathcal{W})$ so that some topologically meaningful relations among those of Defintion 1 are preserved. Of course, an answer to this problem depends on deciding what counts as a map between event structures, i.e., we must provide a category of event structures and event maps. In Thomason (1989), Thomason considers as maps those functions that preserve complete precedence. We, on the contrary, shall take as maps those functions that preserve $\mathrm{R}_{+}, \mathrm{R}_{-}, O$ and also the operations $\oplus, \ominus$ up to equivalence $\equiv$. The philosophical reason for this choice is that these maps preserve just enough structure to be able to model the potential infinite divisibility of time. The mathematical reason is that these maps correspond to the "right" maps on the space of instants on event structures, that is, order-preserving continuous maps. In particular, such maps would allow us to obtain an equivalence of categories that is closely related to that in point-free topology between topological spaces and locales (Johnstone, 1986) or, even closer to the present setting, between topological spaces and formal topologies (Sambin, 2003); see Pinosio (2017). For the present purposes, however, the following suffices:

Definition 17. Let $\mathcal{W}, \mathcal{W}^{\prime}$ be event structures. A function $f: \mathcal{W} \rightarrow \mathcal{W}^{\prime}$ is an event map, or simply a map, if it preserves $O, \mathrm{R}_{+}, \mathrm{R}_{-}$, and, in $G T$, it preserves the operations $\oplus, \ominus$ up to $\equiv$, that is: 


$$
f(a \oplus b) \equiv f(a) \oplus f(b)
$$

and similarly for $\ominus$.

Note that a map must preserve the covering relation $\preceq$, and that because of the Alexandroff correspondence it can be seen in two complementary ways, that is, either as a homomorphism of models of a first-order theory or as a bicontinuous map between bitopological spaces preserving the "tolerance" or "proximity" $O$. We shall often switch between these perspectives in the sequel, but note that these two ways of seeing maps between event structures are not in general equivalent when one takes inverse limits of inverse systems of event structures; see in particular section 8. Furthermore, note that we require preservation of $\oplus, \ominus$ up to the defined equality $\equiv$; we shall discuss this choice in section 8 in relation to the axiom of extensionality.

We can now construct a map $f$ from an event structure $\mathcal{W}$ to a canonical event structure induced by its space of instants $K(\mathcal{W})$ as follows:

DEFINITION 18. Let $\mathcal{W}$ be an event structure and let $S \subseteq W$ be a subset of $W$. We define the left and right endpoints of $S$, denoted as $l(S), r(S)$ for $l(S), r(S) \in K(\mathcal{W})$, by letting:

(1) $l(S)=\bigvee\left\{x \in K(\mathcal{W}) \mid S \subseteq F_{x}\right\}$

(2) $r(S)=\bigwedge\left\{x \in K(\mathcal{W}) \mid S \subseteq P_{x}\right\}$

If $S=\{a\}$ for some $a \in W$, then we abuse our notation and denote $l(\{a\})$ simply as $l(a)$, and similarly for $r(\cdot)$.

The instants $l(S), r(S)$ of Definition 18 are well defined since by Proposition 9 $K(\mathcal{W})$ is a complete lattice. Moreover we have:

Lemma 13. Let $\mathcal{W}$ be an event structure and $S \subseteq W$. Then $l(S), r(S) \in B(\mathcal{W})$ and moreover $S \subseteq F_{l(S)}, S \subseteq P_{r(S)}$.

We can then define a canonical event structure induced by the space of instants on an event structure $\mathcal{W}$ as follows:

DEFINITION 19. Let $\mathcal{W}$ be an event structure and let $K(\mathcal{W})$ be its space of instants. The event structure generated by $K(\mathcal{W})$, denoted as $E(K(\mathcal{W}))$, is defined by letting $W$ be the set

$$
\{U \subseteq K(\mathcal{W}) \mid U \text { is open, nonempty and order-convex }\}
$$

And by defining the event structure relations and operations as in Lemma 2.

It is a routine matter to verify that Definition 19 does indeed yield an event structure satisfying the axioms of GT. Furthermore, the above definition is clearly a generalization of the event structure induced by the unit interval $E(\mathbb{I})$ considered in section 3.3 to the more general class of linearly ordered topological spaces that can be constructed as spaces of instants of an event structure. We can now formulate, however:

Proposition 10. Let $\mathcal{W}$ be an event structure and let $f: \mathcal{W} \rightarrow E(K(\mathcal{W}))$ be defined by letting: 


$$
f(a)=(l(a), r(a))
$$

For any $a \in W$. Then $f$ is an event map, that is, it preserves $\mathbf{R}_{+}, \mathbf{R}_{-}, O, \oplus, \ominus$.

The proposition above answers the question we posed as it allows us to see the events in an event structure $\mathcal{W}$ as intervals of the linear order of instants $K(\mathcal{W})$.

This suggests that one ought to be able to set up a pair of functors between the category of event structures and event maps on one side, and a suitable category of totally ordered topological spaces on the other side. This would improve on the approach in Thomason (1989), which provides functors between a category of event structures and homomorphisms, and a category of linear orders and monotone multi-valued maps. In this sense, it is helpful to note that our event maps can be seen as an instance of the "approximate maps" which have been introduced in Banaschewski and Pultr (2010) as a representation of localic morphisms.

\$7. Instants in the context of GT. The constructions in the previous sections only relied on the axiom system $\mathrm{GT}_{1}$, and to a large extent they could have been carried out even for the weaker $\mathrm{GT}_{0}$. We shall now consider the behaviour of instants in the context of the stronger system GT, and in the presence of further assumptions on the covering relation $\preceq$. In this section, unless specified further, we shall only consider models of GT.

7.1. Minimal events and overlapping classes. In this section we show that the axioms of GT imply that maximal overlapping classes of events are maximal filters in the $\preceq$ preorder, and introduce the concept of a $\preceq$-minimal event, an event that is minimal in the $\preceq$ preorder. This latter notion is of particular interest since under certain assumptions on event structures maximal overlapping classes of events have a canonical presentation as the upset of a $\preceq$-minimal event under the covering ordering $\preceq$, which in turn means that maximal overlapping classes are principal maximal filters. The reader should be aware, however, that we are not extending the ontology of instants, which remains composed only of the notions of boundary and infinitesimal interval.

In order to see that in the context of GT maximal overlapping classes of intervals are maximal filters let us introduce a partial "pseudo-meet" operation $\square: W \times W \rightarrow W$ such that, for any $a, b \in W$ with $a O b$ :

$$
a \sqcap b=(a \oplus b) \ominus b
$$

This operation satisfies all the properties of a meet with respect to the $\preceq$ ordering (see Pinosio (2017, p. 97)) with the exception that (i) it is only partial since it is defined only if $a, b$ overlap, and (ii) it is only a "pseudo-meet", because $a \sqcap b \preceq a, b$ and $a \sqcap b$ is maximal with this property in the $\preceq$ ordering but it is not a maximum, since $\preceq$ is in general a preorder and not a poset.

We can now easily state the following result, whose proof can be found in (Pinosio, 2017, p. 127):

Proposition 11. Let $\mathcal{W}$ be an event structure. Then $A \subseteq W$ is a maximal overlapping class if and only if it is a maximal filter under $\preceq$. 
Hence, in the context of GT maximal overlapping classes are nothing more than maximal filters under the covering ordering $\preceq$. We now introduce the concept of a $\preceq$-minimal event:

Definition 20. Let $\mathcal{W}$ be an event structure and let $\mu \in \mathcal{W}$. We say that $\mu$ is $\preceq$-minimal in $\mathcal{W}$ if

$$
\mathcal{W} \models \forall x(x \preceq \mu \rightarrow \mu \preceq x)
$$

LEMMA 14. Let $\mathcal{W}$ be an event structure, $\mu \in \mathcal{W}$ be a $\preceq$-minimal event, and $x \in$ $B(\mathcal{W})$. Then either $\mu \in P_{x}$ or $\mu \in F_{x}$.

LEMMA 15. Let $\mathcal{W}$ be an event structure, $\mu \in \mathcal{W}$ be a $\preceq$-minimal event, and $a \in W$. Then $a O \mu$ implies $\mu \preceq a$.

We are now interested in considering conditions on event structures so that maximal overlapping classes are always generated as upsets of $\preceq$-minimal events. This is of interest because, as we shall see starting from section 8 , inverse limits of inverse systems of finite event structures are such that maximal overlapping classes are always generated by $\preceq$-minimal events. The most salient condition in this sense is compactness of the topology $\mathcal{A}_{\preceq}$, whose open sets are the $\preceq$-upsets:

Proposition 12. Let $\mathcal{W}$ be an event structure such that $\mathcal{A} \preceq$ is compact; then $A \subseteq$ $W$ is a maximal overlapping class if and only if $A=\uparrow \preceq \mu$ for a $\preceq$-minimal $\mu \in W$.

Hence, if an event structure is such that $\mathcal{A}_{\preceq}$ is compact, then all its maximal filters are principal:

Proposition 13. Let $\mathcal{W}$ be an event structure that is well-founded or such that $\mathcal{A}_{\preceq}$ is compact. Then every maximal filter of $\mathcal{W}$ is principal.

We now turn to the consideration of infinitesimal intervals in the context of GT.

7.2. Infinitesimal intervals in GT. The following is the main result of this section:

THEOREM 4. Let $\mathcal{W}$ be an event structure such that $\mathcal{A}_{\preceq}$ is compact, and let $A \subseteq W$. Then the following hold:

(1) A is a maximal overlapping class if and only if $A=C_{i}$ for $i \in I(\mathcal{W})$

(2) Every infinitesimal interval $i \in I(\mathcal{W})$ is of the form $\left(P_{l(\mu)}, \uparrow \preceq \mu, F_{r(\mu)}\right)$ for $\mu \in$ $W$ a $\preceq$-minimal event.

(3) The set of infinitesimal intervals and the set of maximal overlapping classes are in bijective correspondence

Proof. The right-to-left direction of claim (1) follows from Proposition 6. For the left-to-right direction assume $A$ is a maximal overlapping class. Then by Proposition 12 $A=\uparrow \prec \mu$ for a $\preceq$-minimal $\mu \in W$. Let then $i$ be the boundary $\left(P_{l(\mu)}, \uparrow \prec \mu, F_{r(\mu)}\right)$. To see that $i$ is an infinitesimal interval it suffices to note that $\mu \in P_{l(\mu)} \mathbb{O}$ but $\mu \notin F_{r(\mu)}$.

Claim (2) follows from Proposition 6 and 12 and the left-to-right direction of (1).

For claim (3), let then $j: I(\mathcal{W}) \rightarrow \mathcal{P} W$ be the map defined by letting $j(i)=C_{i}$. Clearly the map is injective, and the left-to-right direction of the claim above shows that the map is surjective.

An important consequence of the previous proposition is that the converse of property (2) of Proposition 6 holds: 
COROLlARY 2. Let $\mathcal{W}$ be an event structure such that $\mathcal{A} \preceq$ is compact or $\preceq$ is wellfounded, and let $i \in K(\mathcal{W})$ be such that $C_{i}$ is a maximal overlapping class. Then $i \in I(\mathcal{W})$ is an infinitesimal interval.

\$8. Unity, universality and limits. We now begin to investigate more thoroughly how Kant's figurative synthesis can be modelled by means of inverse systems and limits of finite event structures. The reader unfamiliar with inverse systems and inverse limits of first-order models of a theory can consult Achourioti and van Lambalgen (2011), where these notions are already employed in a Kantian context. We take as our point of departure the collection of finite models of GT, which we conceive as a formal correlate for Kant's notion of possible "temporal forms" of experience.

In particular, recall that according to Kant if we consider any possible experience of succession of perceptions, we are immediately aware that the judgment regarding the temporal order of such perceptions is merely subjective, unless it is subsumed under a universal rule which makes this succession objective - and, ultimately, able to be communicated. An objective succession then requires subsumption of perceptions under the category of causality. This subsumption, however, requires itself a manifold on which it can be applied, and in particular it requires a temporal intuition which can encompass any possible succession of perceptions - not just actual experiences - and which supports the formulation of judgments of objective temporal succession. The consequences of this objective temporal determination are then fully determined by the properties of this all-encompassing temporal intuition. We provide a formal correlate to the thoroughgoing determination of time as formal intuition with respect to judgments of temporal order by means of Theorem 6 in this Section.

8.1. Inverse systems of finite event structures. The guiding intuitions of the constructions that follow are that "parts of time are times" (A169/B211) and that "different times are only parts of one and the same time." (A31-2/B47). We interpret the former as meaning that there exist finite families of "parts of time", which obey the same axioms as time itself ("are times"). The second quote we understand to mean that there exists a unified time ("one and the same time") which is in some sense universal. In light of these considerations we posit:

DEFINITION 21. Let $\mathcal{W}$ be an event structure and let $\mathcal{W}^{\prime}$ be a submodel of $\mathcal{W}$. An event map $r: \mathcal{W} \rightarrow \mathcal{W}^{\prime}$ is a retraction, and $\mathcal{W}^{\prime}$ is a retract of $\mathcal{W}$, if the restriction of $r$ to $\mathcal{W}^{\prime}$ is the identity.

DEFINITION 22. Let $T$ be a directed partial order. An inverse system of finite event structures indexed by $T$ is a family $\left\{\mathcal{W}_{t} \mid t \in T\right\}$ of finite models of GT together with a family of retractions $\mathcal{F}=\left\{r_{t s}: \mathcal{W}_{t} \rightarrow \mathcal{W}_{s} \mid s, t \in T, t \geq s\right\}$ satisfying:

(1) $r_{s s} \in \mathcal{F}$ is the identity for any $s \in T$

(2) $r_{t s} \circ r_{v t}(a)=r_{v s}(a)$ for any $v, t, s \in T$ with $v \geq t \geq s$

The underlying idea of the above definitions is that inverse systems of finite event structures and retraction maps model acts of the figurative synthesis. A retraction map $r_{t s}: \mathcal{W}_{t} \rightarrow \mathcal{W}_{s}$ can be interpreted as a "refinement" of $\mathcal{W}_{s}$ through the addition of new events by the figurative synthesis, in agreement with the divisibility and extendibility of time. The directedness condition on $T$ models the synthesis of the unity of apperception, since given two possible experiences with temporal forms $\mathcal{W}_{t}, \mathcal{W}_{s}$ it must be 
possible to "synthesize" or "combine" them into a single experience with temporal form $\mathcal{W}_{v}$, of which $\mathcal{W}_{t}, \mathcal{W}_{s}$ are submodels or "parts". It was already argued in Achourioti and van Lambalgen (2011) that the directedness condition provides an accurate "formal correlate" for the synthesis of the unity of apperception. We shall be particularly interested in the inverse limits of such inverse systems in order to obtain a formal correlate to the intuition of time "as an object" (B161n); the requirement of directedness will then be crucial, as it ensures that the inverse limit is well behaved.

Indeed, the question now arises whether the class of all finite event structures, which is a formal correlate to the class of all possible temporal forms of experience that can be produced by the figurative synthesis, can be given the structure of an inverse system. We begin with the following result:

THEOREM 5. Let $\mathcal{W}$ be an event structure and let $\mathcal{W}^{\prime}$ be a finite submodel of $\mathcal{W}$. Then $\mathcal{W}^{\prime}$ is a retract of $\mathcal{W}$.

Proof. Let $\mathcal{W}$ be an event structure and let $\mathcal{W}^{\prime}$ be a finite submodel of $\mathcal{W}$. We can easily construct a retraction map $r: \mathcal{W} \rightarrow \mathcal{W}^{\prime}$ as follows. Fix a universal cover $c \in \mathcal{W}^{\prime}$; for any $a \in \mathcal{W}$ let $m_{p}(a) \in \mathcal{W}^{\prime}$ be an event with the property that $a \mathbf{R}_{-} m_{p}(a)$ and there exists no $b \in \mathcal{W}^{\prime}$ with $a \mathbf{R}_{-} b, b \mathbf{R}_{-} m_{p}(a), m_{p}(a) \mathbb{R}_{-} b$, if such an event exists. Otherwise let $m_{f}(a)=c$. Define $m_{f}$ dually by replacing $\mathbf{R}_{+}$for $\mathbf{R}_{-}$and $\mathbb{R}_{+}$for $\mathbb{R}_{-}$. Let then the map $r: \mathcal{W} \rightarrow \mathcal{W}^{\prime}$ be defined as follows:

$$
\begin{aligned}
& r(a)=a \quad \text { if } a \in \mathcal{W}^{\prime} \\
& r(a)=\left(c \oplus m_{f}(a)\right) \ominus m_{p}(a) \quad \text { otherwise }
\end{aligned}
$$

We show that $r$ so defined is a retraction map. First note that it preserves $\mathbf{R}_{+}, \mathbf{R}_{-}, O$. For instance, if $a \mathbf{R}_{+} b$ then clearly $m_{f}(a) \mathbf{R}_{+} m_{f}(b)$, but then the axioms for $\oplus$ imply that $c \oplus m_{f}(a) \mathbf{R}_{+} c \oplus m_{f}(b)$ and hence that $\left(c \oplus m_{f}(a)\right) \ominus m_{p}(a) \mathbf{R}_{+} c \oplus m_{f}(b) \ominus m_{p}(b)$ and we are done. Furthermore, $r$ preserves $\oplus, \ominus$ up to the defined equality $\equiv$. Indeed, let $a, b \in \mathcal{W}$ be such that $a \oplus b$ is defined. We show that $r(a \oplus b) \equiv r(a) \oplus r(b)$, as the case for $\ominus$ is analogous. Now, by linearity either $a \mathbf{R}_{+} b$ or $b \mathbf{R}_{+} a$. We consider the two cases separately.

If $a \mathbf{R}_{+} b$ then it follows from the axioms for $\oplus$ that $a \equiv a \oplus b$, hence $r(a \oplus b) \equiv$ $r(a) \oplus r(b)$, and we are done.

If $b \mathbf{R}_{+} a$ then $a \oplus b \equiv_{-} a$ and also $b \equiv_{+} a \oplus b$. Then $r(a \oplus b) \equiv_{-} r(a)$, and $r(b) \equiv_{+} r(a \oplus b)$. But then clearly since $b \mathbf{R}_{+} a$ then $r(b) \mathbf{R}_{+} r(a)$, hence by the same reasoning $r(a) \oplus r(b) \equiv_{+} r(b), r(a) \oplus r(b) \equiv_{-} r(a)$, and $r(a) \oplus r(b) \equiv r(a \oplus b)$ follows by transitivity of $\equiv_{+}, \equiv_{-}$.

Thus $r$ is an event map. Moreover, it is clearly the identity on $\mathcal{W}^{\prime}$ by definition, hence it is a retraction.

Note that in the treatment up to this point, and in the proof above in particular, we have not assumed the extensionality axiom stating that:

$$
a \equiv b \rightarrow a=b
$$

Nevertheless, since the discussion of inverse systems and limits of event structures will be simpler if this axiom is assumed, we shall assume it. In its presence, then, an event map preserves $\oplus, \ominus$ up to identity $=$ rather than the defined equality $\equiv$. 
The reader who has philosophical qualms about this axiom in view of section 3.3.6 should note that the whole discussion could be reformulated without this axiom, at the expense of a slightly more involved treatment, in terms of setoids. In particular, the definition of inverse systems provided above, and that of inverse limits, would be modified accordingly; for instance, a thread of the inverse limit would satisfy the coherence conditions of definition 22 up to the defined equivalence $\equiv$ rather than equality $=$, and the results in the present and following sections would carry over entireyl, so that the presence of the extensionality axiom is immaterial and only useful in that it allows for a simpler discussion.

We then have:

LEMMA 16. Let $\mathcal{W}, \mathcal{W}^{\prime}$ be finite event structures that satisfy the same geometric extension of GT. Then there exists a finite event structure $\mathcal{W}^{\prime \prime}$ and retraction maps $g: \mathcal{W}^{\prime \prime} \rightarrow \mathcal{W}, r: \mathcal{W}^{\prime \prime} \rightarrow \mathcal{W}^{\prime}$

Proof. Let $\mathcal{W}, \mathcal{W}^{\prime}$ be such that they satisfy the same geometric extension $\mathcal{G}$ of GT. By means of a "dynamic proof" (Coquand, 2002), we can construct a finite model $\mathcal{W}^{\prime \prime}$ of $\mathcal{G}$ such that $\mathcal{W}, \mathcal{W}^{\prime}$ are submodels of $\mathcal{W}^{\prime \prime}$. By Theorem 5, there are retraction maps $r: \mathcal{W}^{\prime \prime} \rightarrow \mathcal{W}^{\prime}$ and $g: \mathcal{W}^{\prime \prime} \rightarrow \mathcal{W}$.

Thus, the class of finite event structures can be endowed with the structure of an inverse system by simply indexing the finite event structures by a set $T$ and letting $s \leq t$ if there exists a retraction map $r: \mathcal{W}_{t} \rightarrow \mathcal{W}_{s}$; the set $T$ is then directed because of Lemma 16. This presentation of the inverse system of all finite event structures is particularly pleasant since it makes clear that the formal correlate of the figurative synthesis is not the index set of an inverse system of finite event structures but the finite event structures and retraction maps among them. In particular, the inverse system of all finite event structures will be called the finitary spectrum of GT.

We now want to impose further conditions on inverse systems of finite event structure so that we can model the process of potential infinite divisibility, or divisio logica, of the temporal continuum. Intuitively, this can be done by requiring that given any event structure $\mathcal{W}_{t}$ in the system, there exists an event structure that retracts to it and which "divides" any boundary $x \in K\left(\mathcal{W}_{t}\right)$ :

DEFINITION 23. Let $\mathcal{W}, \mathcal{W}^{\prime}$ be event structures such that $\mathcal{W}^{\prime}$ is a submodel of $\mathcal{W}$, let $r: \mathcal{W} \rightarrow \mathcal{W}^{\prime}$ be a retraction map, and let $x \in K\left(\mathcal{W}^{\prime}\right)$. We say that $\mathcal{W}$ splits $x$ if there exists $a, b \in W$ with $a \mathbb{O} b$ such that

(1) $\{a, b\}$ and $r^{-1} P_{x} \cup r^{-1} F_{x}$ are $\mathbb{O}$-apart

(2) $a, b \preceq c$ for any $c \in C_{x}$

While the above definition formulates the concept of "splitting" by mere reference to instants, it is important to note that it captures two distinct philosophical concepts. The first is the concept of "dividing" an event, which occurs if $\mathcal{W}$ is finite and $x \in I\left(\mathcal{W}^{\prime}\right)$. In this case $\mathcal{W}$ introduces two non-overlapping events that are covered by the $\preceq$-minimal event $\mu$ that generates the infinitesimal interval in $I\left(\mathcal{W}^{\prime}\right)$; this $\preceq$-minimal event must exist since $\mathcal{W}^{\prime}$ is finite and thus $\preceq$ is compact. Hence, $\mathcal{W}$ effectively splits this minimal event, thereby splitting any event that covers $\mu$, and thus it splits the infinitesimal interval that $\mu$ generates. The second concept is that of "splitting" or "refining" a boundary, which correspond to Kant's claim that "a boundary can be filled, but in such a way that the series is not indicated": this occurs when $x \in B\left(\mathcal{W}^{\prime}\right)$. Now, we have insisted that 
Kant talks about divisions of parts of time and of boundaries in modal terms. This is to be interpreted as reflecting a notion of potentiality: parts of time can always be subdivided to infinity, and boundaries can always be split, since there are no simple parts in time. We can then express this modal take on infinite divisibility as follows:

DEFINITION 24. Let $\left(T, \mathcal{W}_{t}, r_{v s}\right)$ be an inverse system of finite event structures. We say that it satisfies infinite divisibility, or that is is an infinite divisibility inverse system, if for any $i \in T$ and instant $x \in K\left(\mathcal{W}_{i}\right)$ there exists $j \in T, j \geq i$ such that $\mathcal{W}_{j}$ splits $x$.

Clearly, the finitary spectrum of GT is an infinite divisibility inverse system.

A natural question at this point is whether the inverse limit of an inverse system of finite event structures can itself be regarded as an event structure. This is the case because the axioms of GT are all geometric sentences, and these are preserved to limits of inverse systems (Pinosio, 2017, p. 138). In particular, the inverse limit of the finitary spectrum of GT is an event structure, which we take as a "formal correlate" of time as an "infinitely given magnitude", an "object" or a (formal) intuition (B161n).

8.2. The topology on the limit of inverse systems. Since the limit $\mathcal{V}$ of an inverse system of finite event structures is itself an event structure, we can investigate its topological properties. In particular, we have:

Proposition 14. Let $\left(T, \mathcal{W}_{s}, r_{t s}, \mathcal{V}\right)$ be an inverse system of finite event structures. Then $\mathcal{V}$ has a universal cover.

COROLlary 3. Let $\left(T, \mathcal{W}_{s}, r_{t s}, \mathcal{V}\right)$ be an inverse system of finite event structures. Then op $\left(\mathcal{A}_{\preceq}\right)$ is compact.

PROOF. $o p\left(\mathcal{A}_{\preceq}\right)$ is the Alexandroff topology whose open sets are downsets under $\preceq$; now every open open cover of $\mathcal{V}$ by downsets must contain a downset containing a universal cover, since universal covers exist in $\mathcal{V}$ by Proposition 14. But then this downset is already a cover of the whole space, and we are done.

One might wonder if the topology $\mathcal{A}_{\preceq}$ on an inverse limit $\mathcal{V}$ of an inverse system of finite event structures is also compact, but we shall soon see that the answer is negative. The reader acquainted with inverse limits of topological spaces might be surprised at this fact, since the inverse limit of an inverse system of finite topological spaces is always compact. Indeed, we have:

Proposition 15. Let $\left(T, \mathcal{W}_{s}, r_{t s}, \mathcal{V}\right)$ be an inverse system of finite event structures. Equip $\mathcal{V}$ with the topology $\tau$ having as a basis the family $\left\{\pi_{s}^{-1}(D) \mid s \in T, D \subseteq\right.$ $\left.\mathcal{W}_{s}, D=\downarrow_{\preceq} D\right\}$ of preimages of downsets from $\mathcal{W}_{s}$ for any $s \in T$. Then $\mathcal{V}$ is a compact topological space.

ProOF. Equip every $\mathcal{W}_{s}$ for $s \in T$ with the discrete topology; since the inverse limit of finite discrete topological spaces is always compact, and $\tau$ is a coarsening of this topology on the limit, it is also compact.

The reader should note, however, that we do not compute the limit of an inverse system of finite event structures in the category of topological or bitopological spaces, but in the category of event structures. Hence, the topology $\mathcal{A}_{\preceq}$ on the limit need not coincide with the topology $\tau$ induced on it by taking preimages of downsets under $\preceq$; indeed, in general $\tau$ is coarser than $\mathcal{A}_{\preceq}$ on the limit. 
Now, since in general the topology $\mathcal{A}_{\preceq}$ on the limit $\mathcal{V}$ is not compact, the results in section 7 that rely on the compactness of $\mathcal{A}_{\preceq}$ do not apply to $\mathcal{V}$. In particular, Proposition 12 does not apply, which then means that also Theorem 4 and Corollary 2 do not apply. Still, the question remains whether for limits $\mathcal{V}$ of inverse systems of finite event structures, despite the failure of compactness of $\mathcal{A}_{\prec}$, the correspondence between maximal overlapping classes and $\preceq$-minimal events of Proposition 12 still stands; this would in turn imply that all the other propositions in section 7 also stand. The following result answers this question in the affermative:

PROPOSITION 16. Let $\left(T, \mathcal{W}_{s}, r_{t s}, \mathcal{V}\right)$ be an inverse system of finite event structures. Then $A \subseteq V$ is a maximal overlapping class if and only if $A=\uparrow \preceq \mu$ for a $\preceq$-minimal $\mu \in V$.

Proof. See (Pinosio, 2017, p. 142)

COROLlaRY 4. Let $\left(T, \mathcal{W}_{s}, r_{t s}, \mathcal{V}\right)$ be an inverse system of finite event structures and let $a \in V$. Then there exists $a \preceq$-minimal event $\mu$ with $\mu \preceq a$

Proof. Any singleton set $\{a\} \subseteq V$ can be extended to a maximal overlapping class $A$ using the axiom of choice; by Proposition 16 there must be a $\preceq$-minimal event $\mu \preceq$ $a$.

The above proposition effectively states that the compactness of the topology induced on the limit by the basis of preimages of upsets under $\preceq$ is sufficient to ensure the correspondence between maximal overlapping classes and $\preceq$-minimal events, even in the absence of compactness for the topology $\mathcal{A}_{\preceq}$ on $\mathcal{V}$; we can then use all of the results of section 7 for $\mathcal{V}$.

8.3. Universality of GT. In order to achieve the required universality result for time as intuition we especially consider the finitary spectrum of GT, i.e., the inverse system of all finite models of GT. The Kantian justification for considering this rather special inverse system is that it models the full action of the figurative synthesis. In formal terms, the temporal form of a possible experience is nothing else that a finite model of GT. Thus, the inverse limit of the finitary spectrum of GT models the action of the figurative synthesis in producing, and synthesizing, all possible temporal forms of experience, through which time as an intuition - the limit of the system - is first cognized. Since time as an intuition is the all-encompassing time in which all possible experiences must be able to be determined, this means that the inverse limit of the finitary spectrum should allow us to understand the universality of time as an intuition ensured by the action of the figurative synthesis.

Let then $\left(T, \mathcal{W}_{s}, r_{v s}, \mathcal{V}\right)$ be a countable geometrically complete inverse system of finite event structures, that is, each geometric sentence not derivable from GT is represented by a countermodel in the system by the finite model property of GT; note that the finitary spectrum of GT is clearly such a system. We have:

THEOREM 6. The following hold:

(i) $\mathcal{V} \models G T$

(ii) for any geometric sentence $\varphi, \mathcal{V} \models \varphi$ iff $G T \vdash \varphi$

Hence, the inverse limit of all finite models of GT is a universal model for the theory of GT, and it is then "thoroughgoingly determined" with respect to the schemata of possible temporal judgments, as time as formal intuition should be, in a similar way in 
which Euclidean geometry is "thoroughgoingly determined" since it can be axiomatized by a complete theory having a universal model (Tarski, 1959). This thoroughgoing determination grounds the necessary thoroughgoing determination in time of empirical appearances. Note, moreover, that the inverse limit is universal for $\left(T, \mathcal{W}_{S}, r_{v s}\right)$ is also "unique" in the sense of category theory: any two limits of the inverse system are isomorphic.

\$9. The time continuum as the limit on the finitary spectrum. In this section we investigate the space of instants $K(\mathcal{V})$ on inverse limits of inverse systems of finite event structures and, in particular, of inverse systems satisfying infinite divisibility, such as the finitary spectrum of GT. We shall see that the continuum emerging from this analysis is not quite the real continuum, although it bears to it an interesting relation that will be exploited also in the following chapter. Recall that given any inverse system of finite event structures, not necessarily satisfying infinite divisibility, we immediately obtain that the space of boundaries $B(\mathcal{V})$ on the limit $\mathcal{V}$ is compact Hausdorff because of Corollary 1 in section 5. The full space of instants $K(\mathcal{V})$ on $\mathcal{W}$ is not Hausdorff but only $T_{0}$ in virtue of the infinitesimal intervals that are inserted in the jumps of $B(\mathcal{V})$; it however still compact and is connected (see Theorem 3). Moreover, note that the subspace of boundaries of $K(\mathcal{V})$ is just $B(\mathcal{V})$.

We begin by considering the direct limit construction on inverse systems of finite event structures, which allows us to provide a further correlate to the infinity of time.

9.1. Direct limits. Let $\left(T, \mathcal{W}_{s}, r_{v s}, \mathcal{V}\right)$ be an inverse system of finite event structures. Since any map $r_{v s}: \mathcal{W}_{v} \rightarrow \mathcal{W}_{s}$ is a retraction map this implies that $\mathcal{W}_{s}$ embeds in $\mathcal{W}_{v}$, i.e., there exists a map $e_{s v}: \mathcal{W}_{s} \rightarrow \mathcal{W}_{v}$ which is an embedding; in our case, it is just the identity map. We are therefore entitled to consider the direct system $\left(T, \mathcal{W}_{t}, \sqsubseteq, e_{s v}\right)$ where $\sqsubseteq=o p(\leq)$ is the opposite order of $\leq$, i.e., $t \sqsubseteq s$ iff $s \leq t$ for any $s, t \in T$, and $e_{s v}$ is the identity embedding. For this direct system it is possible to define a direct limit $\mathcal{D}$ in the usual way (see Hodges (1997)). We then have:

LEMMA 17. Let $\left(T, \mathcal{W}_{s}, r_{v s}, \mathcal{V}\right)$ be an inverse system of finite event structures. The direct limit $\mathcal{D}$ of the direct system $\left(T, \mathcal{W}_{s}, \sqsubseteq, e_{s v}\right)$ is a countable model of $G T$, it is isomorphic to a submodel of $\mathcal{V}$, and hence it is a retract of $\mathcal{V}$.

The direct limit $\mathcal{D}$ is isomorphic to the submodel of $\mathcal{V}$ given by all the threads $\xi \in \mathcal{V}$ which become eventually constant, i.e., those threads such that there exists $t^{\prime} \in T$ with $\xi(t)=a$ for all $t \geq t^{\prime}$. We shall abuse our notation and call this submodel of $\mathcal{V}$ also $\mathcal{D}$.

The existence of this simple direct system provides us with a further correlate to the infinity of time.

First, let $\mathcal{W}_{t}, \mathcal{W}_{s}$ be finite event structures such that $\mathcal{W}_{s}$ is a submodel of $\mathcal{W}_{t}$. We say that $\mathcal{W}_{s}$ is future bounded for $\mathcal{W}_{t}$ if there exists $a \in \mathcal{W}_{t}$ such that $b \mathrm{R}_{-} a \wedge a \mathbb{O} b$ for any $b \in \mathcal{W}_{s}$. The notion of past bounded for $\mathcal{W}_{t}$ is defined dually, and if $\mathcal{W}_{s}$ is both past and future bounded in $\mathcal{W}_{t}$ then it is simply bounded. Of course, in the finitary spectrum of GT every event structure $\mathcal{W}_{s}$ embeds into an event structure $\mathcal{W}_{t}$ for which $\mathcal{W}_{s}$ is bounded. Intuitively, this means that any event structure can be extended by adding events to the left and the right of its universal cover, thereby introducing a larger universal cover, and so forth to infinity. Thus, the direct system captures formally the dynamic notion of the potential infinity of time that was informally analyzed, in similar terms but for the case of space, in Friedman (2012). 
Second, the above implies that any finite event structure embeds into the inverse limit $\mathcal{V}$ of the finitary spectrum of GT, i.e., into time as an intuition, even though - most importantly - this inverse limit is not composed out of finite event structures as "parts of time", because it is ultra-connected. Hence, $\mathcal{V}$ is infinite in the sense that "every time is part of the unique, all-encompassing time".

9.2. The space of instants on the limit of inverse systems. We now come to the consideration of the space of instants on the limit of inverse systems of finite event structures, and in particular of inverse systems that satisfy infinite divisibility. The following result shows that the space of instants $K(\mathcal{V})$ on an inverse limit $\mathcal{V}$, not necessarily satisfying infinite divisibility, is separable:

PROPOSITION 17. Let $\left(T, \mathcal{W}_{s}, r_{t s}, \mathcal{V}\right)$ be an inverse system of finite event structures. Then the following hold:

(1) $B(\mathcal{V})$ is separable

(2) $K(\mathcal{V})$ is separable if and only if $B(\mathcal{V})$ has countably many jumps

(3) $K(\mathcal{V})$ is second countable if and only if $B(\mathcal{V})$ has countably many jumps

Proof. See (Pinosio, 2017, p. 148).

The above proposition immediately yields the following corollary:

COROLlaRY 5. Let $\left(T, \mathcal{W}_{s}, r_{t s}, \mathcal{V}\right)$ be an inverse system of finite event structures. Then $K(\mathcal{V})$ is separable if and only if it is second countable.

We now focus our attention on the space of boundaries on limits of inverse systems of finite event structures that satisfy the requirement of infinite divisibility, since these are the culmination of our treatment in this chapter: they provide the Kantian continuum. We begin with the following result:

LEMMA 18. Let $\left(T, \mathcal{W}_{s}, r_{t s}, \mathcal{V}\right)$ be an infinite divisibility inverse system. Then for any $\xi, \xi^{\prime} \in \mathcal{V}$, if $\xi \mathbb{O} \xi^{\prime}$ then there exists $\xi^{\prime \prime}$ between $\xi$ and $\xi^{\prime}$, i.e., $\xi \mathbb{O} \xi^{\prime \prime}, \xi^{\prime \prime} \mathbb{O} \xi^{\prime}$ and $\xi^{\prime \prime} \mathrm{R}_{+} \xi, \xi^{\prime \prime} \mathrm{R}_{-} \xi^{\prime}$. Moreover, $\xi^{\prime \prime}$ can be taken to be a thread $\xi^{\prime \prime} \in \mathcal{D}$.

Proof. Let $\xi, \xi^{\prime} \in \mathcal{V}$ be such that $\xi \mathbb{O} \xi^{\prime}$, and let $s$ be the least index such that $\mathcal{W}_{s}=$ $\xi_{s} \mathbb{O} \xi_{s}^{\prime}$. If there exists an event $a \in \mathcal{W}_{s}$ between $\xi_{s}, \xi_{s}^{\prime}$ we are done, since the eventually constant thread $\gamma$ defined by $a$ will be between $\xi$ and $\xi^{\prime}$ in $\mathcal{V}$. Otherwise there is a boundary $x \in B\left(\mathcal{W}_{s}\right)$ with $\xi_{s} \in P_{x}, \xi_{s}^{\prime} \in F_{x}$; by infinite divisibility then there must be $t \geq s$ and an event $a \in \mathcal{W}_{t}$ such that $a$ is between $\xi_{t}$ and $\xi_{t}^{\prime}$ in $\mathcal{W}_{t}$, and we can take the eventually constant thread defined by $a$ as above.

The result above states a sort of density for event structures, which the limit $\mathcal{V}$ of an infinite divisibility inverse system satisfies: given any two events which do not overlap, a third event can be found in between not overlapping both. We now have:

LEMMA 19. Let $\left(T, \mathcal{W}_{s}, r_{t s}, \mathcal{V}\right)$ be an infinite divisibility inverse system. Then for any $\xi \in \mathcal{D}$, the set $\{\mu \in \mathcal{V} \mid \mu \preceq \xi, \mu \preceq-$ minimal $\}$ is uncountable, and hence $I(\mathcal{V})$ is uncountable.

Corollary 6. Let $\left(T, \mathcal{W}_{s}, r_{t s}, \mathcal{V}\right)$ be an infinite divisibility inverse system. Then $\mathcal{A}_{\preceq}$ on $\mathcal{V}$ is not compact.

PROOF. By Corollary 4 and Lemma 19 the family $\{\uparrow \preceq \mu \mid \mu \in V$ is $\preceq$-minimal $\}$ is an uncountable open cover of $\mathcal{V}$ which does not have a finite subcover. 
9.3. The Kantian continuum as the Alexandroff COTS. We can now provide a general characterization of the space of instants $K(\mathcal{V})$ on the limit of infinite divisibility inverse systems. Since the space of boundaries $B(\mathcal{V})$ on the limit of an inverse system of finite event structures is a compact and separable linear order, we can make use of the characterization of this type of orders which has been given in Ostaszewski (1974).

More specifically, for the total order $B(\mathcal{V})$ where $\mathcal{V}$ is the limit of an inverse system of finite event structures let us define two equivalence relations $\equiv, \sim \subseteq B(\mathcal{V}) \times B(\mathcal{V})$ as follows. We let $x \equiv y$ if the cardinality of the set of points between $x, y$ is countable, where a point $z$ is between $x, y$ if $x \leq z \leq y$ or $y \leq z \leq x$. Clearly this is an equivalence relation, and for simplicity we denote the equivalence class of a point $x \in$ $B(\mathcal{V})$ under $\equiv$ as $\widehat{x}$. We then let $x \sim y$ if $x=y$ or if $\widehat{x}=\{x, y\}$. Note that for any two points $x, y \in B(\mathcal{V})$, if $x \sim y$ and $x \neq y$ then $(x, y)$ defines a jump in $B(\mathcal{V})$, since given any $z \in L$ with $x<z<y, x \equiv y$ implies $x \equiv z$ and this implies that $z \in \widehat{x}$, which yields a contradiction. We denote with $\widetilde{x}$ the equivalence class under $\sim$ of any $x \in B(\mathcal{V})$. These equivalence relations can be used to characterize the total order of boundaries $B(\mathcal{V})$ on the inverse limit $\mathcal{V}$ of an infinite divisibility inverse system as follows:

THEOREM 7. Let $\left(T, \mathcal{W}_{s}, r_{t s}, \mathcal{V}\right)$ be an infinite divisibility inverse system. Then the following hold:

(1) For any $x \in B(\mathcal{V}), x$ has either an immediate predecessor $x^{-}$, or an immediate successor $x^{+}$, but not both.

(2) For any $x, y \in B(\mathcal{V})$, if $y$ has no immediate predecessor and $x<y$, then there are uncountably many boundaries between $x$ and $y$ (and similarly if $y$ has no immediate successor and $y<x$ )

Proof. Let $\left(T, \leq, \mathcal{W}_{s}, r_{t s}\right)$ be an infinite divisibility inverse system, $\mathcal{V}$ be its inverse limit, and let $x \in B(\mathcal{V})$. We first show that $x$ cannot have both an immediate predecessor $x^{-}$and an immediate successor $x^{+}$. Suppose otherwise. Then $\left(x^{-}, x\right),\left(x, x^{+}\right)$ define jumps, and hence (i) $\left\{y \in B(\mathcal{V}) \mid x^{-}<y<x^{+}=\{x\}\right.$. By Lemma 10 there are events $\xi, \xi^{\prime} \in \mathcal{V}$ such that $\xi \in P_{x} \cap F_{x^{-}}$and $\xi^{\prime} \in P_{x^{+}} \cap F_{x}$, hence $\xi \mathbb{O} \xi^{\prime}$. By Lemma 18 then there must be a third event $\xi^{\prime \prime}$ between $\xi$ and $\xi^{\prime}$, and hence by Lemma 9 there are boundaries $z, w$ with $x^{-}<z<w<x^{+}$, which is a contradiction with (i) above. Hence $x$ cannot have both an immediate predecessor and an immediate successor.

We now show that $x$ must have either an immediate predecessor or an immediate successor. Consider then $C_{x}$. Since it is a pairwise overlapping set of events, it can be extended to a maximal overlapping set of events $A$. Hence by Theorem 4, which holds for $\mathcal{V}$ because of Proposition 16, $A=C_{i}$ for an infinitesimal interval $i$ of the form $\left(P_{l(\mu)}, \uparrow_{\preceq} \mu, F_{r(\mu)}\right)$ for $\mu \in V$ a $\preceq$-minimal event. Now, either $i<x$ or $x>i$; without loss of generality assume $i<x$. Then $i$ defines a jump $(z, w)$ in $B(\mathcal{W})$ according to Lemma 11, and in particular $z=l(\mu)=\bigvee\left\{y \in K(\mathcal{V}) \mid \mu \in F_{y}\right\}$; we claim that $l(\mu)$ is an immediate predecessor of $x$, i.e., $x=w=r(\mu)$. Suppose towards a contradiction that $l(\mu)<r(\mu)<x$. Then by Lemma 10 there exists $a \in P_{x} \cap F_{r(\mu)}$, and hence $\mu \mathbb{O} a, \mu \mathbf{R}_{-} a$. It is then straightforward to check that for any $b \in C_{i}, a \preceq b$ because $\mu \preceq b, b O c$ for some $c \in C_{x}$. But then $a \preceq \mu$ which gives a contradiction, and we are done.

To show claim (2) let $x, y \in B(\mathcal{V})$ be such that $x<y$ and $y$ has no immediate predecessor. We first show that there must be an eventually constant thread $\gamma \in \mathcal{D}$ 
with $\gamma \in F_{x} \cap P_{y}$. Indeed, since $(x, y)$ is not a jump there is $z$ with $x<z<y$ and by Lemma 10 there are threads $\xi$, $\xi^{\prime}$ with $\xi \in P_{z} \cap F_{x}, \xi^{\prime} \in P_{y} \cap F_{z}$. Hence by Lemma 18 there must be an eventually constant thread $\gamma \in \mathcal{D}$ between $\xi, \xi^{\prime}$, and hence $\gamma \in F_{x} \cap P_{y}$; by Lemma 19 there must then be uncountably many $\preceq$-minimal events covered by $\gamma$, which yield uncountably many boundaries between $x$ and $y$ by taking $\{l(\mu) \mid \mu \preceq \gamma\}$.

Corollary 7. Let $\left(T, \mathcal{W}_{s}, r_{t s}, \mathcal{V}\right)$ be an infinite divisibility inverse system. Then every $x \in B(\mathcal{V})$ is adjacent to exactly one infinitesimal interval $i \in I(\mathcal{V})$, and $|\widehat{x}|=2$ for any $x \in B(\mathcal{V})$.

Given a limit $\mathcal{V}$ of an infinite divisibility inverse system of finite event structures, we can now endow the set of equivalence classes $\widetilde{B(\mathcal{V})}=\{\widetilde{x} \mid x \in B(\mathcal{V})\}$ with a linear order, by letting $\widetilde{x} \leq \widetilde{y}$ if $x \leq y$ or $\widetilde{x}=\widetilde{y}$. Note that this linear order is still a complete lattice; the join of a subset $S \subseteq \widetilde{B(\mathcal{V})}$, for instance, can be defined as $\widetilde{V_{\widetilde{x} \in S} x}$, and similarly for the meet. We then have:

THEOREM 8. Let $\left(T, \mathcal{W}_{s}, r_{t s}, \mathcal{V}\right)$ be an infinite divisibility inverse system. Then $\widetilde{B(\mathcal{V})}$ is order-isomorphic and hence homeomorphic to the unit interval $\mathbb{I}$.

Again following (Ostaszewski, 1974), we obtain:

COROllary 8. Let $\left(T, \mathcal{W}_{s}, f_{t s}, \mathcal{V}\right)$ be an infinite divisibility inverse system. Then $B(\mathcal{V})$ is order-isomorphic and hence homeomorphic to $\mathbb{I} \times\{0,1\}$ with the lexicographic ordering.

PROOF. The claim follows from Theorem 8 and the main Theorem of (Ostaszewski, 1974).

The results above state that the space of boundaries $B(\mathcal{V})$ on an infinite divisibility inverse system is order-isomorphic to the space obtained from the unit interval $\mathbb{I}$ by splitting every real $r \in \mathbb{I}$ into two points $\left(r_{0}, r_{1}\right)$ that define a jump and taking the lexicographic ordering. Now, since there is a one-to-one correspondence between jumps and infinitesimal intervals (Lemma 11) and infinitesimal intervals are situated in the order of $K(\mathcal{V})$ between the two boundaries that define the corresponding jump (Proposition 9), we have that there is an order-isomorphism $f$ from $K(\mathcal{V})$ to the space $\mathbb{I} \times\{0,1 / 2,1\}$ with the lexicographic ordering; note in particular that $f^{-1}(1 / 2)=I(\mathcal{V})$. However, the order-isomorphism is not a homeomorphism, because the topology on $K(\mathcal{V})$ is not the order topology, but is coarser (See section 6). To turn this order-isomorphism into a homeomorphism of topological spaces we must then consider a coarser topology on $\mathbb{I} \times\{0,1 / 2,1\}$. We then have:

DEFINITION 25. The Alexandroff COTS is the ordered topological space whose underlying set is $\mathbb{I} \times\{0,1 / 2,1\}$, ordered lexicographically, and the topology is that induced by the subbasis of order-open rays of the form $\{((x, i), \leftarrow) \mid x \in \mathbb{I}, i \in$ $\{0,1\}\} \cup\{((x, i), \rightarrow) \mid x \in \mathbb{I}, i \in\{0,1\}\}$. We denote this space by $\mathcal{A}$.

THEOREM 9. Let $\left(T, \mathcal{W}_{s}, f_{t s}, \mathcal{V}\right)$ be an infinite divisibility inverse system. Then $K(\mathcal{V})$ is order-isomorphic and homeomorphic, that is, it is isomorphic in the category of ordered topological spaces, to the Alexandroff COTS $\mathcal{A}$.

PROOF. Corollary 8, along with Proposition 9, give the order-isomorphism; continuity of the order-isomorphism and of its inverse is easily checked. 
We adopt the name "Alexandroff COTS" for the topological space $\mathcal{A}$ in Definition 25 since the topological space $\mathbb{I} \times\{0,1\}$ with the lexicographic ordering is known as the "Alexandroff split interval", as it was originally introduced by Alexandroff to provide an example of a separable but not metrizable space; but $\mathcal{A}$ is also a connected ordered topological space in the sense of (Khalimsky et al., 1990). Since the space of instants $K(\mathcal{V})$ on the limit of an infinite divisibility inverse system is order-isomorphic and homeomorphic to $\mathcal{A}$, we conclude that $\mathcal{A}$ provides the general topological structure of the Kantian continuum itself. Most importantly, $\mathcal{A}$ is (isomorphic to) the space of instants on the finitary spectrum of GT, so that the definition of an infinite divisibility inverse system is quite canonical in that all these inverse systems, and in particular the finitary spectrum, give rise to the same space of instants.

Philosophically, the open points of $\mathcal{A}$, corresponding to the infinitesimal intervals in $I(\mathcal{V})$, represent the inexhaustible "in-between" that Brouwer mentions in Passage (7) of section 2.3. Indeed, the process of divisio logica could, in principle, proceed to the transfinite by splitting the instants further; this is witness to the radical impossibility of exhausting the continuum. There is, however, an unsatisfactory aspect to $\mathcal{A}$, namely, the fact that it is a linear order. Indeed, one might argue that if $i \in I(\mathcal{V})$ represents an infinitesimal "fleeting" time between $i^{-}, i^{+}$then it should not be possible to discriminate the order of succession of $i^{-}, i^{+}, i$. We shall address this point in the next section, in relation to Kant's infinitesimals.

\$10. Infinitesimals. In this section we take a closer look at what we called "infinitesimal intervals", and show that they indeed consist of infinitesimals. To this end, we define a set consisting of both boundaries and events, by

$$
\mathcal{D}:=B(\mathcal{W}) \cup\left\{c \in W \mid \exists x, y \in B(\mathcal{W}),(x, y) \text { a jump, } c \in F_{x} \cap P_{y}\right\} .
$$

The set $F_{x} \cap P_{y}$ is non-empty as a consequence of the existence of minimal events w.r.t. the covering relation, and it is a subset of $C_{i}$ for $i \in I(\mathcal{W})$ the infinitesimal interval that lies between $x, y$ in the order.

We define a preorder $\sqsubseteq$ on the set $\mathcal{D}$ by letting $\alpha \sqsubseteq \beta$ iff $\beta \in P \Rightarrow \alpha \in P$ for all pasts $P$. If $\alpha \in B(\mathcal{W})$, " $\alpha \in P$ " means $P_{\alpha} \subset P$. We then have

Lemma 20. For all $x, y, a, b \in \mathcal{D}$

1. $\sqsubseteq$ is reflexive and transitive

2. $x, y \in B(\mathcal{W})$, then $x \sqsubseteq y$ if $x=y$ or $P_{x} \subset P_{y}$

3. $x, y \in B(\mathcal{W}),(x, y)$ a jump, $a \in C$ with $\left(P_{x}, C, F_{y}\right) \in I(\mathcal{W})$, then $x \sqsubseteq a \sqsubseteq y$

4. $x, y \in B(\mathcal{W}),(x, y)$ a jump, $a, b \in C$ with $\left(P_{x}, C, F_{y}\right) \in I(\mathcal{W})$, then $b \sqsubseteq a \sqsubseteq b$

5. $\sqsubseteq$ is not anti-symmetric

The main theorem of (Ostaszewski, 1974) implies that $B(\mathcal{W})$ can be mapped to the unit interval by a quotient map which identifies the extremities of a jump; let $\sim$ be the associated equivalence relation on $B(\mathcal{W})$. Extend $\sim$ to all of $\mathcal{D}$ by taking the disjoint union $\cong$ of $\sim$ and $=$. $\cong$ preserves $\sqsubseteq$. We may then enrich $[0,1]$ with infinitesimals as follows

DEFINITION 26. $\mathcal{E}:=\mathcal{D} / \cong$.

LEMMA 21. ( $\mathcal{E}, \sqsubseteq)$ is a compact connected Alexandrov topological space which is not $T_{0}$, and which has no cutpoints (the infinitesimals provide the 'glue'). 
At this point it may not yet be clear that an event $a$ caught between the extremities of a jump $(x, y)$ can indeed be viewed as infinitesimal, but informally, the explanation is this: let $r$ be the real corresponding to the equivalence class $\{x, y\} / \sim$, then $r \sqsubseteq a \sqsubseteq r$; for any $s \geq r$, we have $r \sqsubseteq a \sqsubseteq s$, whence the magnitude of $a$ is in a sense 0 . A more formal treatment will be given below. In the remainder of this section we will use $\mathbb{R}$ instead of $[0,1]((0,1)$ being homeomorphic to $\mathbb{R})$, because we need the ring structure of $\mathbb{R}$. $\sqsubseteq$ is modified accordingly.

10.1. Duration. Duration is the archetypical example of a "flowing" magnitude, "since the synthesis (of the productive imagination) in [its] generation is a progress in time" (A170/B212). It turns out, however, that of Kant's three modi of time: simultaneity, succession and duration, the latter tends to conflict with succession considered as linear order. Here's why.

Definition 27. A pseudometric on a space $X$ is a function $\delta(x, y): X \times X \rightarrow$ $[0,+\infty)$ satisying

1. $\delta(x, y) \in[0, \infty)$

2. $\delta(x, y)=\delta(y, x)$

3. $\delta(x, y)=0$ if $x=y^{21}$

4. $\delta(x, y) \leq \delta(x, z)+\delta(z, y)$

5. $\delta(r x, r y)=r \delta(x, y)$ (homogeneity, the property of being the same at every scale)

6. $\delta(r+x, r+y)=\delta(x, y)$ (translation invariance)

DEFINITION 28. If the space $X$ carries a topology $\tau$, one says that $(X, \tau)$ is metrizable if there exists a metric $\delta$ on $X$ such that the set of open balls $B(x, r)=\{y \mid$ $\delta(x, y)<r\}$ generates $\tau$. This can happen only if $\tau$ is Hausdorff. Pseudo-metrizability is defined as before; but the Hausdorff property is no longer implied.

LEMma 22. The linear order $K(\mathcal{W})$, with the topology as defined in Theorem 3, is neither metrizable nor pseudo-metrizable.

ProOF. According to Theorem $3, K(\mathcal{W})$ is a $T_{0}$ compact connected ordered topological space. In the presence of $T_{0}$, a pseudo-metric is a metric. However, if $K(\mathcal{W}), \tau$ were metrizable, it would have to be Hausdorff, but the material on the Alexandroff COTS in the previous section shows it is not.

Informally, this means that some basic open subsets of $K(\mathcal{W})$ cannot be assigned a duration.

10.2. The continuum as a vector space. To make duration possible, we must transform $(\mathcal{E}, \sqsubseteq)$ into a vector space, in which the infinitesimals are independent of the reals. A simplified version of this construction goes as follows. Take any $\epsilon \in \mathcal{E}$ satisfying $\epsilon \sqsubseteq 0 \sqsubseteq \epsilon$; think of $\epsilon$ as an indeterminate $X$. Construct the polynomial ring $\mathbb{R}[X]$; note that we thereby introduce expressions such as $1+X r$ which were not in $(\mathcal{E}, \sqsubseteq)$. Take the quotient $\mathcal{R}:=\mathbb{R}[X] /\left(X^{2}\right)$ of $\mathbb{R}[X]$ by the ideal $\left(X^{2}\right)$. In this structure, $X^{2}=0-$ $X$ is nilsquare - and each element can be written as $q+X r$, for real $q, r$, i.e. as a sum of a real and (scalar multiple) of an infinitesimal. This two-dimensional vector space over $\mathbb{R}$ is the ring of dual numbers. Multiplication is given by

$$
(q+X r)(s+X t)=q s+X(r s+q t)
$$

${ }^{21}$ A metric has 'iff' instead of 'if'. 
hence $\mathcal{R}$ is an algebra over the reals.

The algebra $\mathcal{R}=\mathbb{R}[X] /\left(X^{2}\right)$ cannot be linearly ordered, but carries a preorder $\leq$ which is compatible with the ring operations and leaves the position of the nilsquares undecided.

(1) $x \leq y$ implies $x+z \leq y+z$

(2) $x \leq y, 0 \leq r$ implies $x r \leq y r$

(3) $0 \leq 1$

(4) if $e$ is nilsquare, $0 \leq e \wedge e \leq 0$

(5) if $e$ is nilsquare, $r$ a real, then $e r$ is nilsquare

In a vector space, a (pseudo)metric can be derived from a (semi)norm $\|\bullet\|$, which is determined by the following conditions

Definition 29. 1. $\|x\| \geq 0$; if $x=0,\|x\|=0^{22}$

2. $\|\alpha x\|=|\alpha|\|x\|$ (homogeneity)

3. $\|x+y\| \leq\|x\|+\|y\|$ (triangle inequality)

Definition 30. On $\mathcal{R}$, define the seminorm $\|q+X r\|:=\sqrt{q^{2}+X^{2} \cdot r^{2}}$. As a consequence $\|q+X r\|=\|q\|$ and $\|X r\|=\sqrt{X^{2} \cdot r^{2}}=0$.

LEMMA 23. The seminorm just defined is continuous in the sense that $\alpha \sqsubseteq \beta$ implies $\|\alpha\| \leq\|\beta\|$.

Definition 31. A pseudometric on a space $X$ is a function $\delta(x, y): X \times X \rightarrow$ $[0,+\infty)$ satisying

1. $\delta(x, y) \in[0, \infty)$

2. $\delta(x, y)=\delta(y, x)$

3. $\delta(x, y)=0$ if $x=y$

4. $\delta(x, y) \leq \delta(x, z)+\delta(z, y)$

DEFINITION 32. The semi-norm $\|x-y\|$ defines a homogeneous translation invariant pseudo-metric $\delta$ on $\mathcal{R}$ by putting $\delta(x, y)=\|x-y\|$.

LEMma 24. $\delta$ generates the Alexandroff topology on $\mathcal{R}$ determined by $\leq$.

As an example, consider the closed interval $[1,2]$ which contains $1+r X$ and $2+s X$ for all reals $r, s$, but $\delta(1+r X, 2+s X)=1$ for all $r, s:\|2+s X-(1+r X)\|=\|$ $1+(s-r) X \|=1$. The "instants" 1,2 can thus be viewed as filled ("fat") instants in the Kantian sense, which only give diffuse boundaries around an interval. As Weyl put it

It is due to the essence of time (and not to contingent imperfections in our medium) that a fixed temporal point cannot be exhibited in any way, that always only an approximate, never an exact determination is possible.

This is why the strict linear order on $K(\mathcal{W})$ is not suitable for representing duration and (infinitesimal) persistence. Note however that the two-dimensional numbers used to represent duration do not mean that time is somehow two-dimensional; precisely because the strict linear order $K(\mathcal{W})$ is what it is, one needs two mutually incommensurable types of numbers to quantify it.

${ }^{22}$ A norm satisfies the stronger property: $\|x\|=0$ iff $x=0$. 
Finally, we return to the structure $(\mathcal{E}, \sqsubseteq)$. Employing polynomial rings with uncountably many indeterminates and quotienting, the construction given above shows that $(\mathcal{E}, \sqsubseteq)$ can be embedded as a substructure in $(\mathcal{R}, \leq)$, and hence inherits the pseudometric of $\mathcal{R}$. Interestingly, the construction establishes a close affinity between $(\mathcal{E}, \sqsubseteq)$ and the the geometric line of synthetic differential geometry (Kock, 2006).

The next section discusses a surprising application of these notions to Kant's work on physics.

10.3. Infinitesimals in theMetaphysical foundations of natural science. Infinitesimals provide an important bridge between the CPR and the Metaphysical foundations of natural science (MFNS). At B155n we find:

Motion of an object in space does not belong in a pure science, thus also not in geometry; for that something is movable cannot be cognized a priori but only through experience. But motion, as description of a space, is a pure act of the successive synthesis of the manifold in outer intuition in general through the productive imagination, and belongs not only to geometry but even to transcendental philosophy.

This point is developed further in the first chapter ("Phoronomy") of the MFNS:

In phoronomy, since I am acquainted with matter through no other property but its movability, and may thus consider it only as a point, motion can only be considered as the describing of a space - in such a way, however, that I attend not solely, as in geometry, to the space described, but also to the time in which, and thus to the speed with which, a point describes the space.

Surprisingly, speed, hence differentiation, is treated in the MFNS with a liberal sprinkling of infinitesimals. While discussing the change in velocity of an object projected upward from a point $A$, and reversing direction of motion at point $B$, Kant raises the question whether the object can be said to be at rest at point $B$, and answers affirmatively, with the following argument:

The reason for this lies in the circumstance that the motion [of this object] is not thought of as uniform at a given speed but rather first as uniformly slowed down and thereafter as uniformly accelerated. Thus, the speed at point $B$ is not completely diminished, but only to a degree that is smaller than any given speed. With this speed, therefore, the body would, if it were to be viewed always as still rising ... uniformly traverse with a mere moment of speed (the resistance of gravity here being set aside) a space smaller that any given space in any given time no matter how large. And hence it would absolutely not change its place (for any possible experience) in all eternity. It is therefore put into a state of enduring presence at the same place - i.e., of rest - even though this is immediately annulled because of the continual influence of gravity (i.e., the change of this state). (4:486)

Let $q$ be a smooth function representing the height of the object as a function of time, so that $q^{\prime}$ represents the speed. We have generally for all smooth $f$, for all reals $a, b$, nilsquare $\epsilon$,

$$
f(a+b \epsilon)=f(a)+f^{\prime}(a) b \epsilon
$$


Kant claims that at the turning point $B=q(r)$ the speed is a non-zero infinitesimal representing the 'mere moment of speed'. Thus we cannot equate speed at $B$ with $q^{\prime}(r) b \epsilon$, since this quantity equals $0 .{ }^{23}$ Instead we must expand the expression $q^{\prime}(r+c \epsilon)$ to

$$
q^{\prime}(r+c \epsilon)=q^{\prime}(r)+q^{\prime \prime}(r) c \epsilon=q^{\prime \prime}(r) c \epsilon,
$$

which is a non-zero infinitesimal. This is possible because $q^{\prime}$ is also smooth. Now, even if $c$ goes to infinity, the distance traversed between $r$ and $r+c \epsilon$ is the integral of the infinitesimal-valued function $q^{\prime \prime}(r) c \epsilon$ over an infinitesimal interval, and this is indeed "a space smaller that any given space in any given time no matter how large.". If we equate "possible experience (of motion)" with with a process taking place in real (i.e. non-infinitesimal) time, then "it would absolutely not change its place (for any possible experience) in all eternity. It is therefore put into a state of enduring presence at the same place - i.e., of rest.". We have thus obtained a formalization of Kant's notion of rest, which we already discussed intuitively in section 2.6.

\$11. Conclusions. The first main achievement of this work is that we have provided a mathematical analysis of Kant's temporal continuum that clarifies numerous aspects of this concept. We can summarize these aspects by means of the table in Figure 2, where in the left column we display the informal notions of Kant's theory of the temporal continuum, and in the right column the "formal correlates" to these notions in the mathematical theory.

Note that in Figure 2 we mention the distinction between time as the form of intuition and time as an intuition itself, or time as formal intuition. We do not have the space here to go into more detail regarding this distinction, which appears at B161n in the CPR and has been hotly discussed by many commentators (Onof \& Schulting, 2015), so we refer the reader to (Pinosio, 2017) for its extended discussion in the context of the formal model.

A further point of interest that we cannot explore here is the comparison between our formal theory of the Kantian continuum and work on the formalization of the Aristotelian continuum in (Roeper, 2006) and, more recently, in (Hellman \& Shapiro, 2013); but see (Pinosio, 2017, p. 76) for an argument that our setting captures the Aristotelian notion of point more closely than (Hellman \& Shapiro, 2013).

The second main achievement of the present work is that our analysis of Kant's theory of time allows us to understand what "faculties and functions" must be in place for time to satisfy all the synthetic a priori principles for time mentioned in the TA, i.e., we have a formal version of Kant's transcendental argument for time in the CPR. The existence of time as an object satisfying all the synthetic a priori principles for time (the inverse limit $\mathcal{V}$ of the finitary spectrum of GT and its space of boundaries $K(\mathcal{V})$ ) is dependent on the axioms of GT, which ensure directedness of the finitary spectrum of GT - the synthesis of the unity of apperception - and are in turn justified in terms of the categories. For instance, without the action of the category of substance there would be no covering axiom; without covering axiom directedness of the finitary spectrum of GT would fail;

\footnotetext{
${ }^{23}$ At this point our analysis differs from Michael Friedman's, who in his magisterial (Friedman, 2013, p. 50) assumes the speed equals 0 .
} 


\begin{tabular}{|c|c|}
\hline Informal notion & Formal correlate \\
\hline Time as "form of intuition" & Mere multiplicity without constraints (a set) \\
\hline $\begin{array}{l}\text { Time as "formal intuition", } \\
\text { influence of the understand- } \\
\text { ing on sensibility }\end{array}$ & Graded notion: axiom systems $\mathrm{GT}_{0}, \mathrm{GT}_{1}$ and $\mathrm{GT}$ \\
\hline Part of time & A model of GT \\
\hline $\begin{array}{l}\text { Action of the figurative syn- } \\
\text { thesis }\end{array}$ & Inverse systems of finite event structures \\
\hline $\begin{array}{l}\text { Kantian-Aristotelian tempo- } \\
\text { ral boundaries }\end{array}$ & Two-sided boundaries (section 5) \\
\hline $\begin{array}{l}\text { Indecomposability of time } \\
\text { ("time does not arise } \\
\text { through the positing of its } \\
\text { parts") }\end{array}$ & Ultra-connectedness of event structures (section 4) \\
\hline Infinity of time & $\begin{array}{l}\text { Formal boundaries (section 5.2), directed systems of fi- } \\
\text { nite event structures, limit of the finitary spectrum of GT } \\
\text { ("all times are part of one and the same time", section 9) }\end{array}$ \\
\hline Infinite divisibility of time & Infinite divisibility inverse systems \\
\hline $\begin{array}{l}\text { Synthesis of the unity of ap- } \\
\text { perception }\end{array}$ & $\begin{array}{l}\text { Directedness of the class of finite event structures under } \\
\text { retractions (section 8) }\end{array}$ \\
\hline Time as an object & Inverse limit of the finitary spectrum (section 8.1 ) \\
\hline Unicity of time & $\begin{array}{l}\text { Unicity of the inverse limit of the finitary spectrum up to } \\
\text { isomorphism and its universality (section 8.3) }\end{array}$ \\
\hline $\begin{array}{l}\text { Thoroughgoing determina- } \\
\text { tion of time as an object }\end{array}$ & $\begin{array}{l}\text { Inverse limit of the finitary spectrum as a universal model } \\
\text { (Theorem 6) }\end{array}$ \\
\hline The Kantian continuum & $\begin{array}{l}\text { The Alexandroff COTS } \mathcal{A} \text { (Theorem 9) and the ring of } \\
\text { dual numbers (section 10) }\end{array}$ \\
\hline $\begin{array}{l}\text { Kantian infinitesimals and } \\
\text { flowing magnitudes }\end{array}$ & $\begin{array}{l}\text { Infinitesimal intervals (sections } 6 \text { and 10) and nilpotents } \\
\text { in the ring of dual numbers (section 10) }\end{array}$ \\
\hline $\begin{array}{l}\text { External representation of } \\
\text { time }\end{array}$ & Functions from $K(\mathcal{W})$ to $\mathcal{R}$ (see section 10) \\
\hline
\end{tabular}

FIGURE 2. Informal notions and formal correlates

and the limit of the finitary spectrum would have very different properties, since it would not have a universal cover - in other words, different wholly separate "temporal worlds" would be possible. We have then presented here a mathematically precise account of Kant's transcendental argument for time in the CPR and of the relation between the categories, the synthetic a priori principles for time, and the unity of apperception; the most precise account of this relation to date.

We conclude by mentioning very briefly two further questions to investigate, one in logic and one in cognitive science.

In logic: how can one combine the framework in the present paper with that in (Achourioti \& van Lambalgen, 2011) to obtain a complete formal account of Kant's transcendental logic, space and time? 
In cognitive science: can we identify more precise psychological and neural correlates for Kant's notion of self-affection in the description of a space, and can we use Kant's insights to formulate empirically testable hypotheses on the relation between self-consciousness and metacognitive information on sensorimotor functions, information that is accessible to the cognitive system and is perhaps crucial for the representation of a self that persists in time and is located in space?

\section{References.}

Achourioti, T. \& van Lambalgen, M. (2011). A formalization of Kant's transcendental logic. The Review of Symbolic Logic, 4(02), 254-289.

Alexandroff, P. (1937). Diskrete raume. Mat. Sbornik, 2(3), 501-519.

Arthur, R. T. (1995). Newton's fluxions and equably flowing time. Studies in History and Philosophy of Science Part A, 26(2), 323-351.

Banaschewski, B. \& Pultr, A. (2010). Approximate maps, filter monad, and a representation of localic maps. Archivum Mathematicum, 46(4), 285-298.

Barrow, I. (1976). Lectiones geometricae. Georg Olms Verlag.

Carelli, M. G. \& Forman, H. (2012). Representation of multiple durations in children and adults. Child Development Research, 2011.

Casasanto, D. \& Boroditsky, L. (2008). Time in the mind: using space to think about time. Cognition, 106(2), 579-593.

Casasanto, D., Fotakopoulou, O., \& Boroditsky, L. (2010). Space and time in the child's mind: evidence for a cross-dimensional asymmetry. Cognitive science, 34(3), $387-405$.

Coquand, T. (2002). A completness proof for geometric logic. Computer Science and Engineering Department, University of Gotherburg.

Dehaene, S. \& Brannon, E. (2011). Space, time and number in the brain: searching for the foundations of mathematical thought. Academic Press.

Friedman, M. (2012). Kant on geometry and spatial intuition. Synthese, 186(1), 231255.

Friedman, M. (2013). Kant's construction of nature: a reading of the metaphysical foundations of natural science. Cambridge University Press.

Hellman, G. \& Shapiro, S. (2013). The classical continuum without points. The Review of Symbolic Logic, 6(03), 488-512.

Hodges, W. (1997). A shorter model theory. Cambridge university press.

Johnstone, P. T. (1986). Stone spaces. Cambridge University Press.

Kant, I., Holger, K., Gerresheim, E., Heidemann, I., \& Martin, G. (1908). Gesammelte Schriften. G. Reimer.

Kelly, J. (1963). Bitopological spaces. Proceedings of the London Mathematical Society, 3(1), 71-89.

Khalimsky, E., Kopperman, R., \& Meyer, P. R. (1990). Computer graphics and connected topologies on finite ordered sets. Topology and its Applications, 36(1), 117.

Kock, A. (2006). Synthetic differential geometry. Cambridge University Press.

Kopperman, R., Kronheimer, E., \& Wilson, R. (1998). Topologies on totally ordered sets. Topology and its Applications, 90(1), 165-185.

Longuenesse, B. (1998). Kant and the capacity to judge. Princeton University Press. 
Nicod, J. (2014). Foundations of geometry and induction. Routledge.

Northoff, G. (2012). Immanuel Kant's mind and the brain's resting state. Trends in cognitive sciences, 16(7), 356-359.

Onof, C. \& Schulting, D. (2015). Space as form of intuition and as formal intuition: on the note to b160 in Kant's critique of pure reason. Philosophical Review, 124(1), $1-58$.

Ostaszewski, A. J. (1974). A characterization of compact, separable, ordered spaces. Journal of the London Mathematical Society, 2(4), 758-760.

Palmer, L. (2008). Kant and the brain: a new empirical hypothesis. Review of General Psychology, 12(2), 105.

Palmer, L. \& Lynch, G. (2010). A Kantian view of space. Science, 328(5985), 14871488.

Pinosio, R. (2017). The logic of kant's temporal continuum (Doctoral dissertation, Institute for Logic, Language and Computation, University of Amsterdam).

Roeper, P. (2006). The Aristotelian continuum. A formal characterization. Notre Dame Journal of Formal Logic, 47(2), 211-232.

Rota, G.-C. (1991). The pernicious influence of mathematics upon philosophy. Synthese, 88(2), 165-178.

Russell, B. (1914). Our knowledge of the external world as a field for scientific method in philosophy. Routledge.

Russell, B. (1936). On order in time. Proc. Camb. Phil. Soc. 32, 216-228.

Sambin, G. (2003). Some points in formal topology. Theoretical computer science, 305(1), 347-408.

Tarski, A. (1959). What is elementary geometry? Studies in Logic and the Foundations of Mathematics, 27, 16-29.

Thomason, S. (1984). On constructing instants from events. Journal of Philosophical Logic, 13(1), 85-96.

Thomason, S. (1989). Free construction of time from events. Journal of Philosophical Logic, 18(1), 43-67.

Van Benthem, J. (2013). The logic of time: a model-theoretic investigation into the varieties of temporal ontology and temporal discourse. Springer Science \& Business Media.

van Dalen, D. (2009). The return of the flowing continuum. Intellectica, 1, 51.

Walker, A. G. (1947). Durées et instants. Revue Scientifique, 85, 131-134.

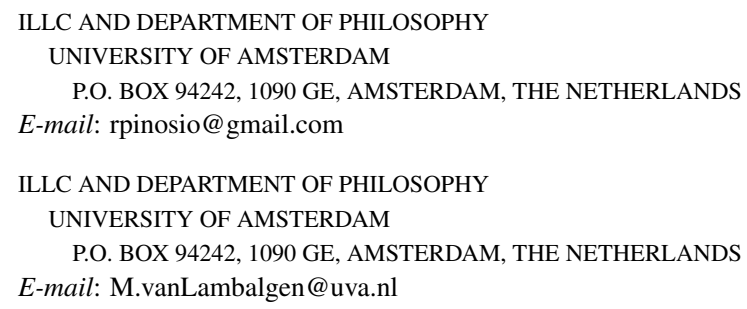

